Meta

Journal des traducteurs

Translators' Journal

\title{
Repères critiques traductionnels français-turc : anaphores pronominales et nominales
}

\section{Tilda Saydi}

Volume 64, numéro 1, avril 2019

URI : https://id.erudit.org/iderudit/1065327ar

DOI : https://doi.org/10.7202/1065327ar

Aller au sommaire du numéro

Éditeur(s)

Les Presses de l’Université de Montréal

ISSN

0026-0452 (imprimé)

1492-1421 (numérique)

Découvrir la revue

Citer cet article

Saydi, T. (2019). Repères critiques traductionnels français-turc : anaphores pronominales et nominales. Meta, 64(1), 20-56.

https://doi.org/10.7202/1065327ar
Résumé de l'article

À l'aide de l'étude traductologique d'un corpus de segments sources en français, traduits vers le turc, le but du présent travail est d'analyser, dans une optique interprétative, la correspondance des anaphores pronominales et nominales en français et leur traduction en turc. Au moyen des notions de procédés et stratégies de traduction, il a été possible de percevoir la fragilité sémantique suscitée par la traduction des anaphores pronominales et la nécessité d'habileté pour leur traduction aussi bien que les aspects traductionnels présentés par les anaphores nominales, tout en soulignant celles de nature pragmatique. Les résultats font valoir le rôle des anaphores pronominales qui n'imitent pas aveuglément les structures grammaticales sources traduites vers le turc, et qui sont en harmonie sémantique avec le texte source. Par ailleurs, les anaphores nominales, bien que leur traduction soit plus proche de leurs équivalents cibles, demandent un savoir-faire de la part du traducteur, qui ne soit pas mécanique, mais interprétatif sur le plan de son art communicationnel.
Ce document est protégé par la loi sur le droit d'auteur. L'utilisation des services d’Érudit (y compris la reproduction) est assujettie à sa politique d'utilisation que vous pouvez consulter en ligne.

https://apropos.erudit.org/fr/usagers/politique-dutilisation/ 


\title{
Repères critiques traductionnels français-turc: anaphores pronominales et nominales
}

\author{
TILDA SAYDI \\ Aydın Adnan Menderes Üniversitesi, Aydin, Turquie \\ tildasaydi@adu.edu.tr
}

\section{RÉSUMÉ}

À l'aide de l'étude traductologique d'un corpus de segments sources en français, traduits vers le turc, le but du présent travail est d'analyser, dans une optique interprétative, la correspondance des anaphores pronominales et nominales en français et leur traduction en turc. Au moyen des notions de procédés et stratégies de traduction, il a été possible de percevoir la fragilité sémantique suscitée par la traduction des anaphores pronominales et la nécessité d'habileté pour leur traduction aussi bien que les aspects traductionnels présentés par les anaphores nominales, tout en soulignant celles de nature pragmatique. Les résultats font valoir le rôle des anaphores pronominales qui n'imitent pas aveuglément les structures grammaticales sources traduites vers le turc, et qui sont en harmonie sémantique avec le texte source. Par ailleurs, les anaphores nominales, bien que leur traduction soit plus proche de leurs équivalents cibles, demandent un savoir-faire de la part du traducteur, qui ne soit pas mécanique, mais interprétatif sur le plan de son art communicationnel.

\begin{abstract}
Using the translational study of a corpus including segments in French, expressed into Turkish, the aim of this article is to analyze for an interpretative purpose, the correspondence of pronominal/nominal anaphors in French with their translation into Turkish. Through notions as translation tools and translation strategies, it has been possible to perceive the semantic fragility provoked by the translation of the pronominal anaphors and the need of skill for their translation between the two languages as well as the translation aspects presented by the translation of the nominal anaphors while underlining those of a pragmatic nature. The results emphasize the role of the target pronominal anaphors which do not blindly imitate the source grammatical structures and which are in semantic harmony with the source text. Moreover, nominal anaphors, although their translation is more similar to their equivalents in Turkish, require the know-how of the translator, in a way which is not mechanical but interpretative in terms of his communicational art.
\end{abstract}

\section{RESUMEN}

Con ayuda del estudio traductológico de un corpus de segmentos fuentes en francés, traducido hacia el turco, el objetivo del presente trabajo es analizar en la óptica interpretativa, la correspondencia de las anáforas pronominales/nominales en francés con su traducción en turco. Por medio de los conceptos de métodos y estrategias de traducción, fue posible percibir la fragilidad semántica generada por la traducción de las anáforas pronominales y la necesidad de habilidad para su traducción entre las dos lenguas así como los aspectos de traducción presentados por las anáforas nominales destacando al mismo tiempo el de naturaleza pragmática. Los resultados hacen valer el papel de las anáforas pronominales que no imitan dado que se tradujo ceguera, hacia el turco, las estructuras gramaticales fuentes y que está en armonía semántica con el texto fuente. Por otra parte, las anáforas nominales, aunque su traducción se vinculó más cerca de sus equivalentes objetivos, piden unos conocimientos técnicos por parte del traductor que no sea mecánico pero interpretativo en cuanto a su arte comunicacional. 


\section{MOTS CLÉS/KEYWORDS/PALABRAS CLAVE}

anaphore pronominale, anaphore nominale, procédé de traduction, stratégie de traduction, français-turc

pronominal anaphor, nominal anaphor, translation process, translation strategy, FrenchTurkish

anáfora pronominal, anáfora nominal, método de traducción, estrategia de traducción, francés-turco

\section{Introduction}

Notre expérience dans le milieu de l'enseignement universitaire ${ }^{1}$, dans des cours de traduction du français vers le turc, mais aussi dans des cours de traduction pédagogique pour l'apprentissage du français langue étrangère en Turquie, nous a mené à identifier l'anaphore comme source de difficulté pour la traduction entre ces deux paires de langue. Ce phénomène de reprise sémantique pose effectivement problème aux futurs traducteurs du français vers le turc. Sans surprise, le problème découle des différences linguistiques entre ces deux langues, lesquelles le locuteur turc doit apprivoiser dans le processus de traduction. Comme l'indique Özçelik:

[i]l est certain que la langue française présente quelques particularités difficiles pour tout apprenant étranger ainsi que pour l'apprenant turc. L'apprentissage de l'écrit, comme tout apprentissage linguistique, place l'apprenant turc devant deux grammaires hétérogènes: la sienne (la grammaire du turc) et celle qu'il doit acquérir (la grammaire du français). S'impose alors à l'apprenant la tâche d'intégrer deux structures différentes, deux langues d'origine différente. (Özçelik 2008: 105)

La notion de grammaire ne se limite pas aux règles théoriques; elle influe également sur la formation du sens, l'interprétation sémantique, la cohérence, le style et l'intelligibilité du message à transférer. Dans cette optique, les pronoms jouent un rôle important dans la construction des anaphores, éléments indispensables, pour le français comme pour le turc, en vue d'assurer cohérence et efficacité dans l'articulation logique du discours. Le français recourt systématiquement à l'anaphore; le turc l'emploie également, mais à sa façon. Il convient de noter que l'anaphore s'appuie à la fois sur les pronoms et sur différentes composantes stylistiques. Dans le cadre du présent travail, nous nous limitons, d'une part, sur le plan grammatical, à l'étude de certaines anaphores pronominales. D'autre part, sur le plan stylistique, nous nous intéressons particulièrement aux anaphores nominales reproduites notamment à l'aide d'un terme générique, d'un synonyme, ainsi que d'une périphrase, pour ce qui est nominatif.

Sur le plan grammatical, il faudra cependant distinguer l'anaphore et le déictique, surtout en raison de leur probable unicité graphique. Le premier tient la place d'un nom, d'un terme ou de sa forme groupale, et ce, par souci de renforcement ou pour éviter la répétition ou la redondance. Au contraire, le second a pour seule vocation de déterminer une unité lexicale préalablement citée en un mot de vocabulaire. Ainsi, l'anaphore joue un rôle important, captivant, voire persuasif; elle s'utilise, à l'écrit et à l'oral, en tant que figure de style. En revanche, le déictique se place près d'un mot, simplement en vue de le désigner, de le qualifier ou d'assurer sa continuité discursive, en fonction de sa fonction grammaticale. À titre d'exemple, le pronom démonstratif composé ceux-là, dans l'énoncé «Parmi les étudiants des langues étrangères, ceux-là 
étudient dans la filière de français langue étrangère», remplit une fonction déictique, signalant une vérité extralinguistique. Au contraire, dans l'énoncé «Des apprenants exceptionnels étudient dans la filière de français langue étrangère; j'aimerais enseigner à ceux-là ", le même pronom démonstratif occupe un rôle singulièrement anaphorique. En effet, il fait référence à une situation linguistique particulière dans un contexte donné.

Du côté stylistique, dans les limites de notre travail, les anaphores portées par un nom générique, par un synonyme ou par une périphrase permettent d'animer l'enchaînement narratif afin de provoquer, d'irriter, d'accentuer ou de reproduire un sentiment, une idée ou une image. Dans un contexte de traduction, notamment entre le français et le turc, ce genre d'anaphores doit être minutieusement analysé pour s'assurer que les anaphores cibles reflètent la même signification et la même nuance que les anaphores sources, sans oublier que «la traduction est une opération qui transpose ce que l'homme pense et non ce que sa langue nomme» (WidlundFantini 2007 : 18).

Dans cette perspective, l'objectif du présent travail est d'illustrer et d'analyser la traduction, du français vers le turc, de certaines anaphores courantes, telles que les anaphores pronominales et nominales. Cela permettra d'égaliser le mieux possible, au niveau traductologique, la continuité, la cohésion et la cohérence intertextuelles, étant donné que ce fait rejoint effectivement les problèmes que la notion de reprise pose dans le processus traductionnel du français vers le turc (et par extension, vice versa), comme en témoigne notre activité pédagogique universitaire avec les apprenants de langues étrangères en Turquie et avec quelques étudiants Erasmus, natifs francophones, apprenant le turc langue étrangère (désormais, TLE). Conformément, d'après Delisle, l'enseignement de la

[...] théorie didactique est comparable à une carte routière qui ne dit pas où il faut aller, mais étale les possibilités. Si le voyageur [pour nous l'apprenant de FLE ou de TLE/le futur traducteur/le futur enseignant/le futur professionnel] dispose d'une bonne carte, il risque moins de se perdre. (Delisle 2005: 117)

Dans cette optique, il apparaît vraisemblablement que le français et le turc possèdent leur flexibilité chacun à leur manière, pour accéder à l'enrichissement lexical et à la diversité langagière, tous deux dans leur propre discursif communicationnel. Conformément aux buts de l'enseignement, à savoir « développer l'aptitude à manier la langue écrite, développer l'esprit d'analyse et la créativité sur le plan linguistique, développer l'aptitude à la traduction des textes pragmatiques et littéraires " (Delisle 2003: 14), la distinction entre le français et le turc sur les genres et le rôle des anaphores est un facteur utile pour ne pas perdre son chemin dans la parité de ces deux langues syntaxiquement non voisines, et le turc ayant largement la capacité d'être elliptique. Pour ce faire, l'examen des segments retirés de l'œuvre littéraire choisie pour ce travail consiste en une approche comparative des anaphores dans une double perspective, soit leurs emplois courants en français et leur reverbalisation, donc leur traduction explicite ou implicite, en turc.

À proprement parler, on définit l'anaphore comme une reprise de ce qui a déjà été annoncé plus tôt dans le texte; elle permet la réutilisation d'une idée ou assure la continuité d'un discours interrompu. En d'autres termes, il s'agit du recommencement, du recouvrement, de la récupération ou de la poursuite de la pensée déjà communiquée. D’ailleurs, l'anaphore est, de prime abord, considérée comme un 
procédé grammatico-lexical qui aide à développer la logique narrative et à enrichir le discursif textuel. Poncharal affirme que

parmi les outils d'appréhension des phénomènes de cohérence, l'anaphore, sous toutes ses formes - anaphores lexicales, fidèles, infidèles, pronominales, associatives, résomptives [...] -, occupe une place centrale. Ce concept a une valeur translinguistique, car il permet de rendre compte des phénomènes de continuité discursive, quelle que soit la langue envisagée. Mais du même coup, il tend à occulter les différences existant entre les langues, quant à leur mode propre de construction de ladite cohérence textuelle. (Poncharal 2010: 1)

En français, les deux catégories d'anaphore qui sont fondamentalement utilisées dans les textes écrits aussi bien que dans le parler, fonctionnent en tant que reprises pronominales et reprises nominales. En outre, comme figure de style, l'anaphore, sous forme de terme générique, de synonyme et de périphrase s'ajoute à ces deux catégories. Dans les limites de cet article, il a paru intéressant d'examiner la traduction du français vers le turc des fragments textuels se rapportant aux anaphores en français, assurées par les pronoms personnel et démonstratif, le terme générique, le synonyme et la périphrase.

\section{Cadre conceptuel, théorique, méthodologique et choix du corpus}

Vinay et Darbelnet (1958/1966: 55) ont recensé sept procédés de traduction: les procédés directs tels que l'emprunt, le calque et la traduction littérale, et les procédés obliques tels que la modulation, la transposition, l'équivalence et l'adaptation. Parmi ceux-ci, nous avons employé la transposition, l'omission (considérée comme un soustype de transposition), l'équivalence et, surtout, la traduction littérale pour traduire des segments choisis contenant des anaphores. Il est vrai que ces procédés ont été critiqués (Ladmiral 1979: 20; Larose 1989: 45; Chuquet et Paillard 1987: 10, cités dans Guidère 2008: 45), car ils ne permettent pas de rendre compte des aspects pragmatique, communicationnel et cognitif du processus de traduction. Cela dit, ceux-ci ont tout de même servi de base pour l'élaboration d'une classification plus large des procédés de traduction. Ballard (2006: paragraphe 5) mentionne d'ailleurs plusieurs travaux qui font référence à ces procédés, comme Chuquet et Paillard (1987), Van Hoof (1989) et Vreck (1992).

Pour pallier ces critiques, nous faisons également appel aux stratégies de traduction, concept complémentaire à celui de procédés. Cela nous permettra de prendre en compte les plans communicatif et pragmatique dans le cadre de la traduction des anaphores nominales. Pour reprendre les paroles de Künzli :

Krings (1986, p. 175) définit les stratégies de traduction comme des plans potentiellement conscients, activés par le traducteur pour résoudre des problèmes de traduction concrets, dans le cadre d'une tâche de traduction concrète. Par exemple, devant un problème de compréhension, le traducteur peut recourir à deux stratégies: soit inférer le sens soit consulter une source d'information. (Künzli 2003: 8-9)

Chesterman (1997: 94-112) classe les stratégies de traduction en deux catégories: les stratégies de compréhension et les stratégies de production. Avant de traduire un texte source, le traducteur s'appuie sur des stratégies de compréhension pour pouvoir décoder le texte dans son esprit. Les stratégies de production lui permettent de reformuler ce qu'il a compris et ainsi de créer le texte le plus approprié possible dans la 
langue cible. D’après Chesterman (1997: 87-92), les stratégies de traduction peuvent être de nature syntaxique, sémantique ou pragmatique. Ces trois niveaux d'analyse nous ont été utiles pour ce travail. Les stratégies syntaxiques nous ont permis de nous pencher sur la structure des phrases et les stratégies sémantiques, sur le sens lexical et textuel des différents éléments de la phrase. Les stratégies pragmatiques nous ont plutôt servi à assurer la transmission de différents types d'information (socioculturelle, pratique, etc.) et aussi à adapter le message au contexte. Elles ont donc été importantes pour la traduction d'anaphores relevant du culturel et de l'abstrait.

Nous nous appuyons ainsi sur ces deux notions, procédés et stratégies de traduction, pour étudier la traduction d'anaphores dans des segments choisis. Plus précisément, décrire les choix traductionnels et analyser les conséquences qui en découlent, tout en étalant le pour et le contre que ces techniques et tactiques peuvent engendrer dans le cadre de la traduction des anaphores en question. Certaines stratégies syntaxiques, sémantiques et pragmatiques deviennent alors fonctionnelles en traduisant les types d'anaphores étudiées. Ainsi, le rôle de la traduction des mots et expressions anaphoriques dans la réussite traductionnelle entre le français et le turc a été mis en lumière.

Cette analyse se base également sur la théorie interprétative de la traduction développée par Seleskovitch et Lederer (2001). Celle-ci décrit les processus cognitifs et la contextualisation textuelle sur lesquels s'appuie l'acte de traduire, ce qui nous paraît nécessaire. Pour Seleskovitch et Lederer (2001: 93), l'opération traduisante comprend trois temps: l'interprétation, la déverbalisation et la réexpression. En précisant qu'il convient de définir la traduction comme un exercice interprétatif, une analyse intelligente du discours, Delisle (1980: 58) ajoute à ces trois temps une phase d'analyse du discours, qui vient en quelque sorte appuyer la compréhension et la contextualisation. Dans le cadre de la théorie interprétative, la déverbalisation constitue l'étape intermédiaire du processus traductif, au cours de laquelle le traducteur déverbalise le sens du texte source pour mieux le réexprimer dans la langue cible.

L'application des procédés de traduction, comme la traduction littérale, la transposition, l'omission et l'équivalence, ainsi que l'utilisation d'autres stratégies de traduction, notamment syntaxiques, sémantiques et pragmatiques, nous permettent de formuler la traduction des anaphores et de montrer leur fonctionnement. Quant au choix des segments, l'œuvre romanesque Les Misérables $(1862)^{2}$ de Victor Hugo nous procure ceux étudiés grâce auxquels nous démontrons la fragilité de l'activité traductionnelle dans le processus de déverbalisation et de réécriture du français vers le turc.

Les textes littéraires, bien qu'ils soient le fruit d'une langue et d'un style soignés et distinctifs, reflètent et exposent tout ce qui peut se passer dans l'univers linguistico-culturel. En outre, Les Misérables, œuvre universelle hugolienne, a été traduit pour la première fois en $\operatorname{turc}^{3}$ en 1862 , juste après sa première publication en français (Yılancıoğlu 2005: 287-296). Depuis, de nouvelles traductions vers le turc ont suivi. Par ses dimensions toujours actuelles et universelles, cette œuvre intemporelle renferme toutes les thématiques dans un véritable trésor de source langagière, toujours contemporaine. C'est pourquoi les énoncés sujets de notre travail retiennent la représentativité anaphorique servant d'objet à nos analyses. Nous ne nous intéressons pas, dans cette étude, aux ouvrages de traduction de l'œuvre publiés vers le turc ni à la critique de ces travaux, mais aux énoncés textuels que l'œuvre comporte et qui 
actualisent à titre d'exemples et en tant que modèles, le fonctionnement des anaphores pronominales et nominales citées plus haut dans l'acte de traduire. Et par conséquent, en parallèle, nous nous intéressons à leur fonctionnement dans l'apprentissage du français ou du turc comme langue étrangère.

Par ailleurs, il nous a été utile de recourir conjointement à des segments littéraires en turc ${ }^{4}$ pour pouvoir vérifier originellement et davantage les caractéristiques des anaphores pronominales et nominales dans la langue turque. Pour ce faire, à chaque fois que nous analysons une anaphore pronominale ou nominale en français et les façons dont celle-ci est traduite vers le turc ${ }^{5}$, nous nous adressons parallèlement à Yaşar Kemal, écrivain universel turc, dont l'ouvrage Çıplak Deniz Çıplak Ada, Bir Ada Hikayesi $4^{6}$, publié en 2012, nous sert de référence afin d'étaler de façon plus visible des exemples comparables renforçant nos analyses.

Qu'il s'agisse des principaux segments en français traduits en turc ou de ceux en turc tirés de l'œuvre de Kemal (2012), auxiliaires à la justification, l'assortiment de l'ensemble des segments repose sur certaines caractéristiques dont ces derniers disposent. Tout d'abord, les segments analysés sont issus d'une même œuvre, donc ils sont en contexte et concernent un ensemble de thèmes. Ensuite, ils sont tous originaires d'un même discours descriptif. Ils s'adressent alors à des destinataires qui sont en définitive les lecteurs finaux natifs (en principe) de la même langue cible. En somme, ils présentent une harmonie cohérente et cohésive du point de vue linguistique et discursif; contextuelle pour ce qui est de la construction de la logique langagière; thématique sur le plan de l'intégralité; et descriptive au niveau du genre textuel. À propos, selon Bordet (2014: 103-119), «la nature des informations que les corpus peuvent fournir pour confirmer ou infirmer les hypothèses de traduction est importante». Nous résumons ici l'affirmation de l'auteur, qui explique que l'utilisation des corpus renvoie à des décisions de traduction qui, à leur tour, soutiennent la transmission efficace du contenu informatif, la production de l'effet rhétorique, la conformité terminologique, phraséologique, discursive aussi bien que la créativité langagière.

Dans cet objectif, nous avons examiné trois segments avec cinq pronoms personnels, quatre segments avec six pronoms démonstratifs et tous anaphoriques, trois segments avec trois anaphores nominales de terme générique, deux segments avec deux anaphores nominales synonymiques et deux segments anaphoriques contenant cinq périphrases. En conséquence, nous avons découvert des traductions possibles en turc qui équivalent auxdits emplois anaphoriques.

\section{Applications et analyses}

En français, un pronom peut servir à reprendre un nom, un groupe de mots ou même une proposition entière. Les pronoms les plus utilisés pour assurer ce genre d'anaphore sont habituellement les pronoms personnels de la troisième personne du singulier et du pluriel - $i l$, elle, ils, elles - les pronoms personnels compléments comme le $(l ')$, la $(l ')$ et les, ainsi que tout pronom démonstratif composé, au singulier et au pluriel, tel que celui-ci/là, celle-ci/là, ceux-ci/là et celles-ci/là. Dans un premier temps, nous étudions les emplois anaphoriques pronominaux, notamment ceux avec les pronoms personnels et démonstratifs. Dans un deuxième temps, nous reprenons successivement les emplois anaphoriques nominaux construits à l'aide de termes 
génériques, de synonymes et de périphrases. Les reprises pronominales et nominales sources sur lesquelles porte l'analyse, et leurs équivalents cibles, sont soulignés. Les phrases originales en français sont suivies de nos traductions en turc, qui, à leur tour, sont suivies par d'autres traductions qui permettent de justifier notre propos. Ces dernières sont faites par différents traducteurs turcs. Elles sont issues des traductions publiées de l'œuvre romanesque Les Misérables de Victor Hugo. Ensuite, des références anaphoriques analogues en turc sont examinées à travers l'ouvrage de Kemal (2012) afin de mieux élaborer la portée de notre travail. Enfin, il convient d'examiner des emplois anaphoriques effectués par des pronoms personnels et leur interprétation en turc.

\subsection{Emploi anaphorique avec pronom personnel}

1) Cosette prit la poupée et la posa doucement à terre avec une sorte de vénération mêlée de désespoir.

(Hugo 1862: 277)

a) Cosette elindeki bebeği kederle karıșık bir saygıyla yere bıraktı.

[Cosette dans sa main la poupée avec de la vénération mêlée respectablement par terre a laissé]

(Hugo 1862/2004: 382-383, traduit par Tunç)

b) Kozet elindek bebeği korku karışan bir saygıyla yere bıraktı.

[Cosette dans sa main de la peur mêlée du respect par terre a laissé]

(Hugo 1862/2003: 62, traduit par Aydoğan)

c) Nos traductions

i. Cosette bebeği alır, hayranlık ve ümitsizlik içinde yavaşça yere bırakır. [Cosette la poupée prend, l'admiration et le désespoir dans lentement par terre laisse]

ii. Cosette bebeği alır ve onu hayranlık ve ümitsizlik içinde yavaşça yere bırakır. [Cosette la poupé prend et la l'admiration et le désespoir dans lentement par terre laisse]

iii. Cosette bebeği alır ve hayranlıkla karışık bir ümitsizlikle onu yavaşça yere birakır.

[Cosette la poupée prend et avec de l'admiration mêlée un désespoir la lentement par terre laisse]

Dans l'exemple 1, le pronom personnel complément la reprend le groupe nominal la poupée; cette anaphore est nécessaire pour l'enchaînement logique de la scène fictive. En 1c, nous fournissons trois propositions de traduction (non exhaustives) pour l'original français. Dans la première proposition (1ci), on peut remarquer l'absence de onu, équivalent turc du pronom personnel complément $l a$, ce qui n'est pas le cas dans les deux autres propositions de traduction. En français, la conjonction de coordination et relie deux propositions; la reprise du complément d'objet direct la poupée par le pronom personnel la procure à l'original une fluidité énonciative. En revanche, la traduction littérale dudit pronom personnel (la par onu) vers le turc n'est pas obligatoire dans la langue cible, ni sur le plan syntaxique ou sémantique. En 1ci, il est clair que l'omission du pronom personnel onu n'entraîne aucune insuffisance ou ambiguïté dans la structure ou dans le sens de la phrase. Comme le signale bien Köse $(2011: 286)$ : 
[1]'emploi des pronoms personnels en turc est assez différent qu'il l'est dans les langues européennes; puisque le turc est une langue agglutinante, l'information sur le sujet de la phrase peut être explicitée à la fois dans la conjugaison verbale et par des pronoms personnels indépendants. C'est pourquoi il est possible de considérer le turc comme une langue qui incise les pronoms. Dans la mesure où le contexte le permet, la reprise du pronom sujet ainsi que du pronom objet n'est pas nécessaire. ${ }^{7}$

Cette souplesse est apparemment due à l'annonce explicite, en turc comme en français, du complément d'objet direct (la poupée, bebeği) dans la première proposition: Cosette prit la poupée, Cosette bebeği alır. La proposition en question est liée à celle qui suit par la conjonction et (ve en turc). La deuxième proposition est anaphorique en ce qui concerne le complément d'objet direct la poupée dans la langue source: [...] et la posa doucement à terre [...]. Quant à la langue cible, il est parfaitement possible de continuer la phrase sans avoir recours au complément d'objet direct exprimé par la (onu). Toutefois, le sujet réel des verbes prit et posa, Cosette, n'est pas exprimé explicitement dans le cas de posa, la deuxième action, et ce, dans les deux langues. Il est donc acceptable d'obtenir en turc un énoncé équivalent à notre première traduction, qui est semblable autant à la traduction de Tunç qu'à celle d'Aydoğan pour ce qui est de l'effacement du pronom personnel complément la (onu). Cependant, en français, une élision semblable est quasiment impossible.

Ainsi, en turc, on peut omettre, sans entraîner de glissement de sens ou de traduction agrammaticale, l'anaphore portée par un pronom personnel complément qui occupe la fonction syntaxique de complément d'objet direct. Bien au contraire, même si ladite anaphore peut être considérée comme facultative, son omission est réfléchie. En effet, en turc, l'anaphore risque le plus souvent d'encombrer la phrase et d'en compromettre l'élégance, ce qui la rend en quelque sorte non idiomatique. En d'autres termes, la présence de l'anaphore risque de rapprocher la traduction de la construction syntaxique du français.

Le prochain exemple va dans le même sens. L'énoncé français présenté dans l'exemple 2 suit immédiatement celui présenté en 1 dans le texte original. Le lien sémantico-logique qui unit ces deux énoncés est exprimé par la présence du pronom personnel anaphorique elle. Dans les traductions turques, ce même lien persiste malgré l'absence d'un pronom anaphorique équivalent.

2) Alors, sans la quitter des yeux, elle joignit les mains [...].

(Hugo 1862: 277)

a) Daha sonra bakışlarını bebekten ayırmadan ellerini birleştirdi [...].

[Ensuite ses regards de la poupée sans séparer ses mains joignirent]

(Hugo 1862/2004: 382-383, traduit par Tunç)

b) Sonra gözlerini bebekten ayırmadan ellerini kavuşturdu.

[Puis ses yeux de la poupée sans séparer ses mains joignirent]

(Hugo 1862/2003: 62, traduit par Aydoğan)

c) Nos traductions

i. Sonra, gözlerini ondan ayırmadan ellerini kavuşturur.

[Puis, ses yeux d'elle sans séparer ses mains joignirent]

ii. Sonra, gözlerini bebekten ayırmadan ellerini kavuşturur.

[Puis, ses yeux de la poupée sans séparer ses mains joignirent]

iii. Sonra o, gözlerini bebekten ayırmadan ellerini kavuşturur.

[Puis elle, ses yeux de la poupée sans séparer ses mains joignirent] 
iv. Sonra o, gözlerini ondan ayırmadan ellerini kavuşturur.

[Puis elle, ses yeux d'elle sans séparer ses mains joignirent]

Les quatre propositions de traduction (2c) sont équivalentes quoique, encore une fois, non exhaustives. Les deux premières traductions (2ci et 2cii) omettent l'équivalent turc du pronom elle, qui reprend le nom propre sujet Cosette dans l'énoncé français; celles-ci adoptent donc une forme plutôt elliptique. Toutefois, la conjugaison du verbe kavușturur (traduisant joignit), le prédicat de la proposition principale, indique nettement que son sujet est Cosette, présent dans la phrase qui précède. Bien que l'équivalent turc du pronom personnel elle puisse être omis dans la traduction, ce n'est pas le cas du le pronom personnel complément correspondant à la, comme le montre nos quatre propositions de traduction. Dans la troisième (2ciii) et la quatrième (2civ) proposition de traduction, le pronom la est traduit littéralement en turc par le pronom o/ondan. Dans la première et la deuxième, le pronom la n'est pas rendu par un pronom équivalent en turc; il est plutôt remplacé par son antécédent, bebekten (correspondant à la poupée). D’ailleurs, les deux traductions publiées (2a et 2b) illustrent ces deux cas de figure, soit l'effacement du pronom personnel complément ou son remplacement par son antécédent.

La troisième et la quatrième proposition de traduction constituent en quelque sorte des calques de la langue source, pour ce qui est de l'utilisation d'un pronom personnel pour exprimer le sujet commun de la proposition principale et de la subordonnée: le pronom personnel elle est rendu en turc par le pronom personnel o. Or, en turc, la présence de o est superflue. En effet, ce type d'anaphore pronominale n'est utile en turc que si l'énonciateur souhaite renforcer volontairement son propos. En fait, l'emploi du pronom personnel o comme sujet réel du verbe principal crée plutôt un embarras lexical, qui conduit à une complexité sémantique. Deux raisons nous motivent à fournir des propositions de traduction non idiomatiques comme 2ciii et 2 civ. D'une part, c'est pour mieux montrer qu'une traduction littérale de l'anaphore pronominale n'est pas nécessaire ou même souhaitable entre le français et le turc. D'autre part, cela permet d'entrevoir la surcharge lexicale que ces pronoms peuvent engendrer dans le texte cible, ainsi que l'abstention sémantique qu'ils génèrent sur le sens, qui se solde par une double anaphore inutile en turc, alors qu'il n'y en a aucune en français.

En turc, la terminaison du verbe indique la personne et le nombre auxquels il est conjugué. Ainsi, les pronoms personnels sont en quelque sorte subsumés dans les terminaisons verbales. C'est pourquoi il est préférable d'omettre les pronoms personnels sujets dans nos traductions des exemples 1 et 2 : la langue turque permet l'identification implicite du sujet du verbe. $\operatorname{Alan}^{8}$ (2005: 343) rappelle que: «En français, l'existence explicite des pronoms en tant que sujet de la phrase est fixe; il faut apprendre aux apprenants (turcs) que ces pronoms sont ineffaçables [...]». Dans une perspective didactique et contrastive, il faut donc rappeler aux apprenants turcophones et francophones les différences dans l'emploi des pronoms personnels sujets qui existent entre ces deux langues. Plus précisément, il ne faut pas essayer de les traduire à tout prix du français vers le turc, comme le confirme Şimşek ${ }^{9}$ :

Les énonciations elliptiques occupent une place importante dans la syntaxe du turc. Notamment, les phrases contenant des sujets apparents et qui sont absentes dans plusieurs autres langues, sont des particularités d'énonciation typiquement propres à la 
langue turque. [...] Le français ne se permet pas ce type d'usage. Le sujet y est obligatoire dans une phrase simple en règle et prend place au début de la proposition. (Şimşek 1987: 7-8)

Dans cette optique, pour répondre aux principes de fonctionnement les plus communément admis de la langue cible, le discursif du turc exige, notamment dans nos exemples, l'effacement du pronom personnel elle. Cela simplifie le discours et renforce son intelligibilité, en plus d'éviter l'encombrement par des mots structuralement et sémantiquement inutiles, sans jamais nuire au message d'origine. Ainsi, il est beaucoup plus pratique et approprié de traduire finalement les exemples 1 et 2 par: Cosette bebeği alır, hayranlık ve ümitsizlik içinde yavaşça yere bırakır. Sonra, gözlerini bebekten ayırmadan ellerini kavușturur. De trois pronoms en français, on passe à un seul en turc. Donc, en turc, pour des phrases qui ont un sujet commun et qui se succèdent, l'effacement des pronoms personnels contextuels devient, la plupart du temps, inévitable. Cela ne compromet pas la cohérence et la cohésion textuelles si le destinataire a accès à l'intégralité du texte cible et donc au contexte immédiat.

Exposons un autre cas du même genre:

3) Sois humain. Il faut avoir pitié des animaux. Et, prenant à son fils le gâteau, il le jeta dans le bassin.

(Hugo 1862: 669)

a) İnsanca davran. Hayvanlara merhamet etmek gerekir. Ve oğlunun elindeki çöreği alıp havuza attı.

[Humainement agit. Aux animaux pitié avoir il faut. Et de son fils dans la main le gâteau en prenant dans le bassin jeta]

(Hugo 1862/2017: 574, traduit par Volkan Yalçıntoklu)

b) İyi yürekli ol. Hayvanlara acımak gerek, dedi. Oğlunun elinden çöreği alıp havuza att1.

[Ait bon cœur. Aux animaux avoir pitié il faut il dit. De son fils de la main le gâteau en prenant dans le bassin jeta]

(Hugo 1862/2015: 458, traduit par Cenap Karakaya)

c) İnsancıl ol. Hayvanlara acımak lazım. Ve oğlundan çöreği alarak havuza attı. [Humain sois. Aux animaux avoir pitié il faut. Et de son fils le gâteau en prenant dans le bassin jeta]

(Notre traduction)

Ces trois traductions montrent comment les pronoms personnels anaphoriques $i l$ et $l e$ s'effacent en turc. Pour traduire le pronom personnel $i l$, sujet réel commun aux deux verbes d'action prenant et jeta, aucun pronom personnel n'est utilisé en turc. La terminaison du verbe attı (jeta), conjugué à la troisième personne du singulier du passé composé, révèle l'identité de son sujet. Quant au complément d'objet direct commun à ces deux verbes, çöreği (gâteau), une fois énoncé pour le premier verbe alarak/alıp (prenant), il n'est pas répété pour le deuxième verbe attı (jeta). Les éléments de la phrase s'enchaînent et permettent la compréhension sans aucune ambiguïté.

Les exemples $(4,5,6$ et 7$)$ ci-après, tirés de l'œuvre de Kemal, nous permettent d'illustrer davantage l'omission idiomatique du pronom personnel en turc, ainsi que la répétition obligatoire des mots en tout terme, qui peut être casuelle, mais sans jamais recourir aux pronoms personnels pour raisons stylistiques et pour le bon effet discursif. 
4) Karanlık kavuşuncaya kadar şu yumuşak ottur, bu değildir, diye kucak kucak ot topladılar, çardağa götürdüler [...].

[La nuit tombe jusqu'à ce que cela douce de l'herbe est, ceci pas, un tas d'herbes

(ils) recueillirent, à la tonnelle (ils) amenèrent]

(Kemal 2012: 331)

a) Jusqu'à ce que la nuit tombe, ils recueillirent un tas d'herbes qu'ils jugèrent douces ou non, les amenèrent sous la tonnelle.

(Notre traduction)

On trouve dans la traduction française 4a les pronoms personnels ils (sujet) et les (complément d'objet direct). Le texte source 4 ne contient pas de pronoms personnels correspondants.

5) Gün doğar doğmaz kuytulara koştular, türlü iri mantarlar topladılar, tuzlayıp közlediler.

[Le jour se lève dès que aux ténèbres (ils) coururent, des espèces de grands champignons (ils) recueillirent, salèrent grillèrent]

(Kemal 2012: 33)

a) Dès l'aube, ils coururent vers les ténèbres, recueillirent espèces de grands champignons, les salèrent, les grillèrent.

(Notre traduction)

De façon semblable, dans l'exemple traduit 5a, le pronom personnel sujet ils et les deux pronoms personnels compléments d'objet direct les n'ont pas d'équivalent dans la phrase source 5 .

6) Uzaktan o kuşun sesi geldi. Çoktandır o kuşun sesini duymuyordu.

[De loin de cet oiseau-là la voix parvint. Depuis longtemps de ce oiseau-là sa voix ne l'entendait pas]

(Yaşar Kemal 2012: 35)

a) Le chant de cet oiseau parvint lointainement. Ça faisait longtemps qu'il ne l'entendait pas.

(Notre traduction)

Dans l'exemple 6, aucun pronom personnel sujet n'est lié au verbe duymuyordu, la terminaison verbale $-\underline{u}$ indiquant la troisième personne du singulier. Dans notre traduction (6a), au contraire, le verbe n'entendait pas (duymuyordu) est accompagné du pronom personnel sujet $i$. De plus, le syntagme o kușun sesi (chant de cet oiseau), sujet du verbe geldi (parvint) dans la première phrase de l'exemple 6, est repris intégralement dans la deuxième phrase, cette fois-ci à titre de complément d'objet direct du verbe duymuyordu. Cette répétition peut être rhétorique (par ex. emphatique), mais elle peut également être motivée par un souci d'éviter toute confusion sémantique quant à l'identité du complément d'objet direct. Dans la traduction 6a, le syntagme chant de cet oiseau est plutôt repris anaphoriquement dans la deuxième phrase, à l'aide du pronom personnel l' $(l e)$.

7) Zehra kovayı aldı koşarcasına çeşmeye gitti, bomboş kovayla geriye döndü. Eve varınca, kovanın boş olduğunun farkına vardı, geri çeşmeye koştu, kovayı musluğun altına koydu. 
[Zehra le seau prit comme si courrait à la fontaine alla, entièrement vide avec le seau rentra. A la maison quand arrivée, du seau vide que était s'aperçut, à la fontaine recourut, le seau du robinet sous posa]

(Kemal 2012: 36)

a) Zehra prit le seau, se rendit à pas précipités à la fontaine; rentra le seau entièrement vide. Dès sa rentrée, elle s'aperçut qu'il était vide, y courut encore, le posa sous le robinet.

(Notre traduction)

De même, on trouve dans l'exemple 7 quatre déclinaisons (kovayı, kovayla, kovanın, kovayı) du nom kova (seau), dont trois occupent la fonction de sujet ou de complément d'objet direct pour un verbe donné: kovay1 [COD] $\rightarrow$ ald 1 (prit); kovanın [sujet] $\rightarrow$ boș olduğunun vardı (était vide); et kovayı [COD] $\rightarrow$ koydu (posa). Cette répétition est atténuée dans la traduction française (7a), alors que kovanın et kovayı sont traduits par les pronoms personnels $i l$ et le, respectivement. Pareillement, dans la traduction 7a, Zehra, le sujet réel des deux phrases de l'exemple 7 est repris anaphoriquement par le pronom personnel elle dans la deuxième phrase. On ne trouve pas de pronom personnel correspondant dans le texte source. De même, le complément circonstanciel de lieu çeșmeye (à la fontaine), qui se rapporte au verbe gitti (se rendit) dans la première phrase de l'exemple 7 , est repris intégralement dans la deuxième phrase. En français, il peut être repris anaphoriquement par le pronom personnel complément $y$, comme dans notre traduction (7a).

Analysons, en outre, un segment avec pronom démonstratif composé.

\subsection{Emploi anaphorique avec pronom démonstratif}

Les exemples 8 et 9, qui suivent, correspondent à des phrases consécutives dans le texte source.

8) L'historien des mœurs et des idées n'a pas une mission moins austère que l'historien des évènements.

(Hugo 1862: 539)

a) Uygarlığın yüzeyinde kalanları, taht mücadelelerini, prenslerin doğumlarını, kralların evliliklerini, savaşları, meclisleri, kamuya mal olmuş önemli şahsiyetleri, devrimleri inceleyen bir olay tarihçisinin [...] bir gelenek ve düşünce tarihçisinden daha saygın bir görev üstlendiğini düşünmüyoruz.

[De la civilisation sur la surface ceux qui restent, trônes de batailles, des princes les naissances, des rois les mariages, les guerres, les assemblées, au peuple appartenant importants les personnalités, les révolutions examinant évènement un historien de [...] une tradition et que l'idée de l'historien plus respectable un devoir qu'il remplisse ne pensons pas]

(Hugo 1862/2017: 296, traduit par Yalçıntoklu)

b) Örf ve adetler ve fikirler tarihçisinin görevi, olaylar tarihçisinin görevinden daha az ciddi olamaz.

[Des mœurs et des idées de l'historien le devoir, des évènements de l'historien que le devoir moins important ne peut être]

(Hugo 1862/2015: 191-192, traduit par Karakaya) 
c) Geleneklerin ve fikirlerin tarihçisinin görevi olayların tarihçisinden daha kolay değildir.

[Des traditions et des idées de l'historien le devoir des évènements que l'historien plus facile n'est pas]

(Notre traduction)

9) Celui-ci a la surface de la civilisation [...], l'autre historien a l'intérieur, le fond [...].

(Hugo 1862: 539)

a) Gelenek ve düşünce tarihçisinin [...] yeraltı katmanlarına inmesi gerekir.

[La tradition et l'idée de l'historien [...] souterrains aux membranes que (il) descend il faut]

(Hugo 1862/2017: 296, traduit par Yalçıntoklu)

b) Olayların tarihçisine düşen uygarlığın yüzeyidir (...). Öteki tarihçiye düşen ise içe ait olandır, temeldir [...].

[Des évènements à l'historien ce qui appartient de la civilisation la surface est (...). L'autre historien ce que (il) a à l'intérieur ce qui appartient c'est]

(Hugo 1862/2015: 191-192, traduit par Karakaya)

c) Nos traductions

i. Olayların tarihçisi, uygarlığın yüzeydeki kısmını ele alır [...]; diğer tarihçi içini, derinliğini açığa çıkarır [...].

[Des évènements l'historien, de la civilisation sur la surface la partie examine [...]; l'autre historien l'intérieur, la profondeur découvre [...].]

ii. O, uygarlığın yüzeyini [...], diğeri derinliğini ele alır [...].

[Lui, de la civilisation la surface [...], l'autre la profondeur examine]

L'exemple 9 commence par le pronom démonstratif celui-ci, qui correspond au sujet du verbe avoir et qui reprend le syntagme historien des évènements dans la phrase précédente (exemple 8). Dans notre traduction 9ci, proposition parmi d'autres, la traduction en turc du sujet ne se fait pas par un pronom démonstratif équivalent, mais plutôt par une reprise intégrale de l'antécédent du pronom démonstratif: olayların tarihçisi (historien des évènements). Cela est nécessaire pour éviter la confusion entre les deux pôles de la comparaison, historien des mours et des idées (geleneklerin ve fikirlerin tarihçisi) et historien des évènements (olayların tarihçisi). En d'autres termes, l'anaphore pronominale source, portée par un pronom démonstratif composé, a été transposée en anaphore nominale dans la langue cible, par la répétition du syntagme olayların tarihçisi (historien des évènements); on omet donc de traduire le pronom démonstratif source.

Cette transposition, on le verra, est nécessaire. En effet, si le traducteur traduit littéralement, et aveuglément, les catégories grammaticales, il provoque une erreur de sens dans son texte cible, comme l'illustre notre traduction 9cii, qui entraîne sciemment un faux sens. Au contraire, la transposition de l'anaphore pronominale source en anaphore nominale cible permet une identification juste de l'antécédent. C'est d'ailleurs la stratégie adoptée par les deux traducteurs du texte source (9a et 9b), Yalçıntoklu et Karakaya.

Comme le constate bien Wolfram (2006: 333), «if structure is at the heart of language, then variation defines its soul». C'est justement pour cette raison qu'il ne faut pas traduire littéralement le sujet réel de l'exemple 9 (celui-ci) dans sa forme pronominale, en employant le pronom démonstratif équivalent en turc (o) comme en 9cii. Cela engendrerait un «effet de rupture» (Kleiber 1994: 90), qui serait dû à 
un faux calcul inférentiel. La traduction fautive 9cii est correcte sur le plan grammatical et syntaxique. Cependant, une interprétation juste de sa sémantique référentielle demande un effort de la part du lecteur, voire une analyse détaillée. Le pronom o dans la traduction 9cii reprend geleneklerin ve fikirlerin tarihçisi (historien des mours et des idées). Or, il devrait reprendre olayların tarihçisi (historien des évènements) pour un accord correct entre le sujet et le verbe. Il s'ensuit qu'une traduction juste et idiomatique ne repose pas que sur une maîtrise de la grammaire et de la syntaxe de la langue cible, mais également sur des connaissances pragmatico-discursives. Ainsi, cela confirme que la connaissance d'une langue ne suffit pas pour la traduire. Selon Seleskovitch et Lederer,

[n] ombreuses sont les raisons pour lesquelles il est impossible à la traduction de se limiter à la seule connaissance des langues. Nous citerons par mémoire la polysémie des mots, l'ambiguïté des phrases, le caractère elliptique des énoncés, la différence de connotation que des mots identiques éveillent dans des domaines différents, les différences stylistiques qui caractérisent les discours dans chacun des domaines de l'activité humaine. (Seleskovitch et Lederer 2007: 153)

Comme la comparaison entre les exemples analysés le démontre, en français, les pronoms s'utilisent très fréquemment dans les énoncés. Ils ont pour mission d'alléger la phrase, de reprendre leur antécédent et d'assurer le maintien du sens le long de la chaîne énonciative.

En développant les supports de l'identité référentielle comme le pronom démonstratif dont la problématique traductionnelle s'explique d'après notre exemple plus haut, Kleiber (1989: paragraphes 22-23) explique la dénotation et la fonction qu'elle se charge de remplir. En effet, la dénotation référentielle comme accomplie par un pronom démonstratif n'est pas un simple renvoi. Pour Kleiber,

[i]l faut encore s'intéresser aux raisons syntaxiques, sémantiques, narratives [...]. Ce problème nous semble fondamental dans la mesure où il touche à la construction cohésive des textes, à leur organisation et progression référentielle. (Kleiber 1989: paragraphes 22-23)

C'est pour cette raison qu'à l'égard de ces mêmes pronoms en turc, bien que ceux-ci remplissent théoriquement les mêmes fonctions grammaticales et expressives qu'en français, leur traduction vers le turc exige une considération particulière. Il y a des moments où ils doivent être introduits, même s'ils ne sont pas sollicités, parce qu'ils sont déjà présents implicitement en général par la suffixation verbale. De surcroît, cet effacement, presque anodin, s'avère parfois dangereux, car il y a encore des moments où l'effacement est interdit, par souci de s'approprier une fausse référence, un antécédent inadéquat. Dans ce cas, à l'inverse du français, il est pratiquement normal de recourir à l'anaphore, autrement dit de répéter dans son ensemble le référé. Plus précisément s'impose «la nécessité de recourir à un composant sémantique plus étoffé» (Kleiber 1990: 241). En prenant ceci en compte, «l'idée fondamentale est que les seules données linguistiques ne suffisent pas à donner le réfèrent visé [...]» (Kleiber 1990: 241), ce qui est parfaitement valable pour le turc, dans une approche sémasiologique:

[L]a perspective sémasiologique parce qu'elle privilégie la spécificité de chaque expression et parce qu'elle motive une volonté d'expliquer unitairement les emplois souvent très différents d'une même forme, amène à des considérations sémantiques 
dans lesquelles la vocation référentielle des marqueurs analysés occupe une place très restreinte. (Kleiber 1990: 242)

De même, avec les pronoms démonstratifs, «la dimension référentielle est, par la force des choses, première, mais, saisie dans la problématique plus générale des symboles indexicaux» (Kleiber 1986), elle a tendance elle aussi à s'éloigner de la réalité que représentent les différents types d'emplois démonstratifs (Kleiber 1990: 242).

À cet égard, les segments justificatifs suivants s'exposent ci-dessous :

10) Comme le temps passe! Nous avons été bien heureux. C’est fini.

(Hugo 1862: 798)

a) Zaman nasıl da geçiyor. Çok mutluyduk. Ama artık bitti.

[Le temps comme passe. Très heureux nous étions. Mais maintenant fini]

(Hugo 1862/2017: 835, traduit par Yalçıntoklu)

b) Günler nasıl da akıp gidiyor! İkimiz çok mutluydık. Artık bitti...

[Les jours comme s'écoulent! Nous deux très heureux on était. Maintenant fini...]

(Hugo,1862/2004: 835 traduit par Tunç)

c) Zaman nasıl da hızlı geçiyor! Çok mutlu olduk biz. Bitti artık.

[Le temps comme rapidement passe! Très heureux avons été nous. Fini maintenant]

(Notre traduction)

La disparition du pronom démonstratif source c' apparaît clairement dans les traductions, notamment dans celles de Yalçıntoklu et de Tunç ainsi que dans la nôtre.

11) L'homme suivi arriva à cette petite colline et la doubla, de sorte qu'il cessa d'être aperçu par l'autre. Celui-ci, ne voyant pas, n'était pas vu, il en profita pour abandonner toute dissimulation et pour marcher très rapidement.

(Hugo 1862: 703)

a) Takip edilen adam bu tepeciğe yaklaşıp arkadan gelenin göremeyeceği şeklide arkaya dolandı. Takip eden adam göremediği için görülemeyeceği düşüncesiyle gizlilik kurallarını bir tarafa bırakıp hızla yürümeye başladı.

[Suivi l'homme à cette petite colline en s'approchant en arrière celui qui arrivait qu'il ne puisse voir de sorte derrière est passé]

(Hugo 1862/2017: 642, traduit par Yalçıntoklu)

b) İzlenen adam bu sözünü ettiğimiz yere geldi, orayı geçti, Böylece öbürü onu göremez oldu, göremediği için kendisinin de görülmediğini bilerek gizlenmeyi bırakıp hızlı hızlı yürümeye başladı.

[Suivi l'homme cet dont on parlait à endroit est venu, le a passé. Ainsi l'autre le ne pouvait voir plus, ne pouvait voir parce que lui-même non plus ne pouvait être vu en sachant à se cacher a renoncé rapidement à marcher s'est mis]

(Hugo 1862/2015: 519, traduit par Karakaya)

c) İzlenen adam bu küçük tepeye varıp onu aştı ve arkasından gelen kişi tarafından görünemez oldu. Arkasından gelen adam artık ne görüyor ne de görülüyordu; bunu düşünerek hızlı hızlı yürümek amacıyla gizlenmekten vazgeçti.

[Suivi l'homme cette petite à colline en arrivant la a passé et de derrière arrivant la personne par invisible a été]

(Notre traduction) 
Notre traduction (11c) comme celles de Yalçıntoklu (11a) et de Karakaya (11b), exposent comment le turc dévoile le pronom démonstratif source celui-ci par l'explicitation ajoutée au texte arkasindan gelen adam dans 11c, ou bien takip eden adam (11a), ou encore öbürü dans (11b).

12) Il reconnaissait que l'une de ces idées était nécessairement bonne, tandis que l'autre pouvait devenir mauvaise; que celle-là était le dévouement et que celle-ci était la personnalité [...].

(Hugo 1862: 126)

a) $\mathrm{Bu}$ düşüncelerden birinin her zaman için doğru olduğunun, diğerinin ise yozlaşmaya müsait olduğunun; biri özveriyi temsil ederken diğerinin benliği temsil ettiğinin [...] farkına varıyordu.

[Ces de pensées l'une toujours pour vraie qu'elle soit, l'autre et à la corruption convenable qu'elle soit; l'une la sacrifice alors que représentant l'autre la personnalité que représentait [...] s'apercevait]

(Hugo 1862/2017: 273, traduit par Yalçıntoklu)

b) Bu iki düşünce [...], birinin doğru olduğunu, diğerinin bozulabileceğini anlıyordu. Biri özveriydi, İkincisi bencillikti [...].

Ces deux pensées [...], de l'une vraie était, l'autre que pouvait corrompre (il) comprenait. L'une le sacrifice était, l'autre l'égoïsme [...].]

(Hugo 1862/2004: 217-218, traduit Tunç)

c) $\mathrm{Bu}$ iki düşünceden birinin mecburen doğru olduğunu diğerinin ise yanlış olabileceğini kabul ediyordu; biri fedakarlık diğeri ise karakter özelliğiydi [...]. [Ces deux de pensées l'une obligatoirement vraie que était l'autre quant à fausse pouvait être (il) acceptait; l'une le sacrifice et l'autre caractère de particularité était $[\ldots]$.

(Notre traduction)

Dans cet exemple, les pronoms démonstratifs sources typiquement français cellelà et celle-ci, faute d'attachement de référence très complexe et ne pouvant être anaphorisés de la même façon en turc, ont été traduits, respectivement par: 12a) biri [une] et diğerinin [autre]; 12b) biri [une] et ikincisi [deuxième]; et 12c) biri [une] et diğeri [autre].

13) Les deux petits pauvres regardèrent venir «ce monsieur» et se cachèrent un peu plus. Celui-ci était un bourgeois.

(Hugo 1862: 669)

a) İki küçük zavallı «mösyönün » geldiğini görünce iyice saklandılar. Bu adam bir burjuvayd.

[Deux petits pauvres «du monsieur » qu' il arrivait quand (ils) ont vu un peu plus se sont cachés. Cet homme un bourgeois était]

(Hugo 1862/2017: 572, traduit par Yalçıntoklu)

b) Zavallıcıklar, bu adamın gelişini izleyip biraz daha saklandılar. Adam bir burjuvayd.

[Les petits pauvres de cet homme l'arrivée en regardant un peu plus se sont cachés. L'homme un bourgeois était]

(Notre traduction)

De plus, dans les deux traductions de l'exemple 13, le sujet de la deuxième phrase (celui-ci) est nettement personnifié, étant traduit par bu adam (13a) ou par adam 
(13b). La fidélité traductionnelle à la catégorie grammaticale du pronom démonstratif source celui-ci inciterait en turc un effort inutile de compréhension, renvoyant au sujet réel de la phrase. Dépendamment des sujets réels ou apparents des phrases précédentes enchaînées successivement, cela risque de provoquer une confusion, de sorte que le destinataire prenne une personne pour quelqu'un d'autre lors de la lecture. En conséquence, force est de constater que la traduction des pronoms démonstratifs anaphoriques du français vers le turc exige une décision habile en vue d'éviter des écrits inadaptés ou sémantiquement énigmatiques dans la langue cible.

En supplément, ci-après, les segments originaires de la littérature turque démontrent la manière dont les sujets réels sont anaphorisés en turc, sans jamais se servir d'un pronom démonstratif, contrairement à ce qui serait normalement fait en français.

14) Kötü bir adam İsmail'i ölüme terk etmiş. [...]. Baban sağ olsaydı Kazdağındaki arkadaşlarını getirir o kötü adamları öldürtürdü.

[Méchant un homme İsmail pour mort a laissé [...]. Ton père en vie s’il était du mont Ida ses amis aurait emmené ces méchants hommes aurait fait tuer]

(Kemal 2012: 227)

a) İsmail avait été laissé pour mort par un homme méchant. [...]. Pour faire tuer celui-ci, ton père, s'il était en vie, aurait emmené ses amis montagnards du mont Ida.

(Notre traduction)

15) Konuşacağı hiç kimsesi yoktu, bir tek evli ablası vardı [...]. Ablası onu çok severdi [...].

Pour parler personne (il) n'avait, seulement mariée sa grande sœur il y avait [...]. Sa grande sœur le beaucoup aimait [...]]

(Kemal 2012: 227)

a) Il n’avait personne à qui parler sauf sa grande sœur mariée [...]. Celle-ci l'aimait beaucoup [...].

(Notre traduction)

16) Atlı oğlanı bekledi. Atlı oğlan beklediğinden çabuk geldi.

[Le chevalier (il) a attendu. Le chevalier qu'il espérait plus vite est arrivé]

(Yaşar Kemal 2012: 231)

a) Elle attendit le chevalier. Celui-ci arriva plus vite qu'elle l'espérait.

(Notre traduction)

Dans les segments sources 14, 15 et 16, il est apparemment remarquable que les anaphores se manifestent telles que jumelles de leur référence. Dans le premier cas (14), figure ainsi un effet de soulignement dû à la répétition en tous termes. Dans les deuxième (15) et troisième cas (16), l'auteur préfère toujours la même méthode, tout en échappant à l'emploi des pronoms démonstratifs, qui, si utilisés, auraient indubitablement un air mal placé et seraient fort susceptibles d'être insignifiants, par opposition à nos traductions), s'assouplissant typiquement par l'intermédiaire des pronoms démonstratifs.

À l'instar de la fragilité traductionnelle des anaphores pronominales, la traduction des anaphores nominales peut, dans certains cas, nécessiter l'ajout des pronoms en turc, bien que ceux-ci soient absents du texte source en français. Par ailleurs, le 
terme générique, la synonymie et la périphrase sont à considérer soigneusement dans la traduction avec les anaphores nominales.

\subsection{Emploi anaphorique nominal}

De multiples anaphores nominales apparaissent par leur devoir de désigner différemment ce qui est déjà évoqué. Elles se distinguent des anaphores pronominales par leur caractère agrammatical. En d'autres termes, elles ne sont pas vides de sens et sont toujours pertinentes, même sans la reconnaissance des mots auxquels elles se substituent. Nous analysons ici trois types d'anaphores nominales, la première accomplie par un terme générique, la deuxième par un synonyme, la troisième par une périphrase. Le choix de ces trois procédés s'explique par la probable complexité spécifique de formulation causée par leurs traductions du français vers le turc.

\subsubsection{Anaphore nominale par terme générique}

L'anaphore nominale de terme générique est constituée par le lexique ordinaire de la langue, à l'aide d'un nom ou groupe de noms qui sert à décrire, à commenter, à argumenter ou à juger le terme ou les mots qu'elle remplace, autrement dit qu'elle connote: «En effet, l'anaphore s'attache aux entités non nommées ou aux entités du deuxième et du troisième ordre, que sont les situations, les évènements et les propositions» (Perdicoyanni-Paléologou 2001: 72).

17) Le 6 juin 1832, une compagnie de gardes nationaux de la banlieue [...] se fit [...] décimer rue de la Chanvrerie. Le fait [...] a été constaté par l'instruction judiciaire ouverte à la suite de l'insurrection de 1832).

(Hugo 1862: 661)

a) 6 Haziran 1832 günü, Banliyöden gelen (...) bir ulusal muhafız bölüğü [...] Chanvrerie sokağında ölüme yollanmıştı [...] bu olay Haziran 1832 ayaklanmasından sonra yapılan adli soruşturma kayıtlarında yer almıştı. [Le 6 juin 1832 du jour, de la banlieue venant (...) une nationale gardes de compagnie [...] Chanvrerie dans la rue à la mort avait été envoyée [...] cet évènement juin 1832 de l'insurrection après fait judiciaire l'instruction dans les enregistrements avait pris place.]

(Hugo 1862/2017: 557, traduit par Yalçıntoklu)

b) 6 Haziran 1832 'de [...] bir banliyö muhafız müfrezesi [...] Chanvrerie sokağında kendini ölüme attı. Bu olay [...] 1832 isyanından sonra açılan adli soruşturmada saptanmıştır.

[Le 6 juin 1832 [...] une banlieue gardes de compagnie [...] Chanvrerie dans la rue à la mort s'était jetée. Cet évènement [...] 1832 de l'insurrection après ouverte à l'instruction judiciaire avait été constaté]

(Hugo 1862/2015: 444, traduit par Karakaya)

c) Nos traductions:

i. 6 Haziran 1832 'de, [...] banliyö ulusal birliklerinden biri [...] Chanvrerie sokağında katledildi. Olay [...] 1832 ayaklanmasından sonra açılan adli soruşturmada ortaya çıkarıldı.

[Le 6 juin 1832, [...] banlieue nationales des compagnies l'une [...] Chanvrerie dans la rue a été assassinée. L'évènement 1832 de l'insurrection après ouverte judiciaire à l'instruction a été découvert] 
ii. 6 Haziran 1832 'de, [...] banliyö ulusal birliklerinden biri [...] Chanvrerie sokağında katledildi. Bu olay [...] 1832 ayaklanmasından sonra açılan adli soruşturmada ortaya çıkarıldı.

[Le 6 juin 1832 [...] banlieue nationales des compagnies l'une [...] Chanvrerie dans la rue a été assassinée. Cet évènement [...] 1832 de l'insurrection après ouverte dans l'instruction a été constatée]

La première proposition indépendante de l'exemple (17) nous renseigne sur un évènement. La deuxième proposition commence par le complément d'objet direct le fait (olay dans les traductions turques) lié au verbe et, par extension, au sujet apparent de la phrase passive. Ce complément d'objet direct est formé par une anaphore nominale, c'est-à-dire lexicale, et reprend l'évènement en le résumant notamment en un seul terme. Dans notre traduction 17ci, l'anaphore nominale est traduite littéralement par le même procédé le fait (olay) tandis que dans 17cii, l'ajout de l'adjectif démonstratif bu (ce) au terme générique est constatable. Donc, puisqu'en turc, les déterminants de catégorie d'articles n'existent pas, en 17ci, le fait est traduit littéralement par olay, étant le nom commun sans article. Différemment, dans 17cii le fait est traduit par bu olay. Ladjectif démonstratif bu (ce) détermine cette fois-ci olay (le fait) dans l'objectif de développer l'évolution de la pertinence textuelle. En conclusion, le déterminant source le devient, par transposition, l'adjectif démonstratif cible bu et le fait revêt sa nouvelle forme ce fait (bu olay) en turc.

En outre, éviter cet adjectif démonstratif est possible mais facultatif, car cela dépendrait strictement des conditions sémantiques. Ainsi, il est possible que la traduction d'une anaphore en terme générique en français soit opérée vers le turc par l'addition d'un tel déterminant ou adjectif démonstratif, par crainte de ne pouvoir mettre suffisamment en relief l'évènement cité. Cette formule, tributaire des textes et contextes donnés, prouve l'utilité de compléter le terme générique par un adjectif démonstratif, inexistant dans l'énoncé source. Par ailleurs, il faudra préciser que l'option de la traduction littérale de l'anaphore authentique, donc du terme générique, ne détruit pas exactement le sens de l'énoncé traduit. Cependant, cette littéralité, et par conséquent l'absence de l'adjectif démonstratif en turc, peut causer, pour la compréhension du destinataire, la perte du fil dans la succession descriptive. De ce fait, pour effectuer une meilleure traduction qui assouplisse et facilite la lecture, tout en lui accordant le même contenu et les mêmes significations sources, l'anaphore nominale qui évoque en un mot ou un terme récapitulatif tout un évènement, nécessite souvent le soutien d'un déterminant tel qu'exposé en (c), comme l'ont formulé les traducteurs Yalçıntoklu 1/3(T1) et Karakaya 1/3(T2). Pour conclure, l'anaphore nominale sous forme de terme générique propose donc une recette traduisante pour la langue d'arrivée, tout comme elle évite normalement la répétition dans le texte de départ. Cela nous permet de penser qu'ici, pour les deux langues,

on trouve dans les chaînes de référence autre chose que des termes de singuliers. On sait par exemple que des groupes nominaux à interprétation dite "générique " peuvent être repris par des termes subséquents ou bien des énoncés entiers. (Corblin 1995: Chapitre 7, les chaînes de référence naturelles)

Des exemples traductionnels d'autres énoncés du même ordre sont identifiables:

18) Ils vivaient, n’ayant plus de noms, désignés seulement par des numéros et en quelque sorte faits chiffres, baissant les yeux, baissant la voix, les cheveux coupés, sous le 
bâton, dans la honte. Puis son esprit retombait sur les êtres qu'il avait devant les yeux. Ces êtres vivaient, eux aussi, [...].

(Hugo 1862: 315)

a) İsimlerini kaybetmiş, kimlikleri sadece numaralarla rakamlarla belirlenmiş, saçları traş edilmiş bir halde gözlerini öne eğip seslerini alçaltarak utanç içinde sopa yiyerek yaşıyorlardı. Ardından, şimdi gördüğü insanları düşünüyordu. Başlarını öne eğip alçak sesle konuşan bu insanlar da [...].

[Leur nom perdu, leur identité seulement avec des numéros avec des nombres indiquée, leurs cheveux rasés d'une façon leurs yeux baissant devant leur voix baissant la honte dans étant battus vivaient-(ils). Ensuite, à présent qu'il voit les hommes (il) pensait. Leur tête baissée devant, avec la voix basse qui parlaient ces gens aussi $[\ldots]$

(Hugo 1862/2017: 668, traduit par Yalçıntoklu)

b) İsimsizdiler, yalnız numaralarıyla çağrılıyorlardı, adeta rakamlardan ibarettiler; gözlerini indirerek,seslerini kısarak, saçları kesik, sopa altında, utanç içinde yaşıyorlardı. Sonra zihni, şimdi gözleri önünde bulunan insanlara dönüyordu. Bu insanlar da saçları kesik, [...] yaşıyorlardı.

[Sans nom (ils) étaient, seul avec leur numéro (ils) étaient appelés, comme si que des nombres (ils) ne se composaient; leurs yeux en baissant, leur voix en baissant, leur cheveux coupés, le bâton sous, la honte dans (ils) vivaient. Ensuite son esprit, à présent ses yeux devant qui se trouvaient avec les gens tournait. Ces gens aussi leurs cheveux coupés [...] vivaient]

(Hugo 1862/2015: 660-661, traduit par Karakaya)

c) Onlar artık isimsiz, sabıka kodu verilmiş, hep yere doğru bakan, sesleri çıkmayan, kafaları traş edilmiş, sırtlarında sopa, utanç içinde yaşıyorlardı. Sonra gözlerinin önündeki yaratıkları düşündü. Bu insanlar da öyle yaşıyorlardı. [Ils, à présent sans nom, criminel de code attribué, toujours vers le sol regardant, leur voix ne s'entendant pas, leur tête rasée, sur leur dos le bâton, la honte dans vivaient. Ensuite de ses yeux devant les créatures il a pensé. Ces hommes aussi comme ça vivaient]

(Notre traduction)

Le sujet ces êtres de l'exemple source (18), anaphore nominale par terme générique, est interprété de la même façon dans les trois traductions vers le turc, par le terme générique cible bu insanlar englobant le sujet de la description, situé dans la phrase énonciatrice.

19) Dieu est derrière tout, mais tout cache Dieu. Les choses sont noires, les créatures sont opaques. Aimer un être, c'est le rendre transparent.

(Hugo 1862: 510)

a) Herşeyin arkasında Tanrı vardır, herşey Tanrıyı gizler. Nesneler siyahtır, yaratıkların rengi donuktur. Bir varlığı sevmek onu şeffaflaştırmaktır.

[De toute chose derrière Dieu il y a, toute chose cache Dieu. Les objets noirs sont, des créatures la couleur est opaque. Un être aimer le rendre transparent c'est]

(Hugo 1862/2017: 238, traduit par Yalçıntoklu)

b) Herşeyin arkasında Tanrı vardır, ama herşey Tanrı’yı gizler. Eşya simsiyahtır, yaratıklar 1şık geçirmezler. Bir varlığı sevmek onu şeffaflaştırmaktır.

[De toute chose derrière Dieu il y a mais toute chose Dieu cache. L'objet tout noir est, les créatures la lumière ne laissent pas passer. Un être aimer le rendre transparent c'est]

(Hugo 1862/2015: 131, traduit par Karakaya) 
c) Tanrı herşeyin arkasındadır ama herşey de Tanrı’yı gizler. Maddeler kapkaradır içleri gözükmez; yaratıklar mat ve donuktur. Bir varlığı sevmek onu saydam hale getirmektir.

[Dieu de toute chose derrière est mais aussi toute chose Dieu cache. Les substances tout noires sont là-dedans invisibles; les créatures opaques et ternes. Un être aimer le transparent rendre c'est]

(Notre traduction)

Le terme générique cible les choses, qui renvoie au mot tout, en d'autres termes aux objets, articles, dispositifs non vivants, est traduit vers le turc par maddeler (notre traduction), nesneler (Yalçıntoklu) ou encore eşya (Karakaya) qui désignent la classification générale des matières inertes. On constate que la méthode de reprise suit la même logique sémantique et le même procédé référentiel dans les langues source et cible. Toutefois, les solutions lexicales des trois traducteurs se rapprochent d'un certain littéralisme dans la langue cible, bien qu'aucune d'elles ne soit en fait la traduction littérale en turc du terme générique source les choses. Cependant, toutes les trois profitent de «la distance dans la proximité» (Ricœur 2004: 52), au sens d'un côtoiement logique de voisinage sémantique. Le terme générique source les choses, s'il est interprété par les traducteurs comme terme symbolisant les substances concrètes, représente «une dualité [pour nous, notifiant un rapport de mutualité] de sens qui est particulièrement caractéristique du symbole» (Ricœur 1965: 21).

D'une manière auxiliaire, découlant de la littérature turque, les segments qui suivent $(20,21$ et 22$)$ confirment l'utilisation du terme générique comme moyen de répétition en turc, soit par l'accompagnement d'un pronom démonstratif, soit à l'aide d'un terme substitutif.

20) Arsen Usta önce değirmenin kanatlarını takacak [...]. Son değirmeni çalıştıracak. $\mathrm{Bu}$ işler çok para istiyor.

[Arsen maître d'abord du moulin les ailes va monter [...] Le dernier moulin (il) va faire travailler. Ces travaux beaucoup d'argent demandent]

(Kemal 2012: 180)

a) Maître Arsen va d'abord monter les ailes du moulin. [...]. Il va faire marcher le dernier moulin. Ces travaux demandent beaucoup d'argent.

(Notre traduction)

21) Efeler efesi Hançerli Efe eski ve kahraman arkadaşının ülkemizi dünya aleme gösterdiğinden dolayı çok sevinecektir [...]. Biz bu ülkeyi böyle yiğitler sayesinde yokken var ettik.

[Des héros le héros Hançerli Efe vieux et courageux de son ami notre pays à l'univers entier qu'il eut montré parce que très heureux sera [...] Nous ce pays de tels héros grâce à tandis que inexistant avons fait exister]

(Kemal 2012: 194)

a) Le grand héros «Hançerli Efe» serait très content que son ancien ami, brave et courageux, montre notre pays au monde entier. C'est grâce à de tels héros que nous, nous avons établi sans aucun moyen, ce pays.

(Notre traduction)

22) Şu suların arasında insanların gelip görmedikleri yerde, dünyaların öteki ucunda bu kızları kara sevda gelip buldu [...]. Bu gurbet elde senden başka sırtlarını dayayacakları kimse yok. 
[Ces eaux entre les hommes ne viennent et ne voient à l'endroit où, des mondes l'autre à bout ces filles l'amour impossible est venu a trouvé [...].Ce lointain dans pays toi sauf sur leur dos à qui s'appuyer personne il n'y a]

(Kemal 2012: 200)

a) L'amour impossible retrouva ces filles, au sein de ces eaux à cet endroit oublié, isolé, à l'autre bout du monde [...]. Dans ce lointain pays étranger, elles n'ont que toi et personne d'autre à qui elles pourront faire confiance.

(Notre traduction)

Les exemples 20, 21 et 22 sont tous illustrés des termes génériques soulignés, localisés dans le deuxième énoncé du segment relié, suivant le premier énoncé qui abrite la description, le portrait ou l'image se rapportant au terme générique. Dans l'exemple 20, bu işler (ces travaux), anaphore par terme générique, contient son explication değirmenin kanatlarını takacak (va monter les ailes du moulin), son değirmeni çalıștıracak (va faire marcher le dernier moulin) dans le premier énoncé. Le terme générique de l'exemple 21 böyle yiğitler (de tels héros) est expliqué juste avant sous la forme eski ve kahraman arkadașının (son ancien ami brave et courageux). Enfin, dans le segment source 22, l'anaphore par terme générique bu gurbet elde (dans ce lointain pays étranger), a son déchiffrement entier insanların gelip görmedikleri yerde, dünyaların öteki ucunda (à cet endroit oublié, isolé, à l'autre bout du monde) dans l'énoncé qui le devance.

En français, les mots ou termes peuvent également être repris par des synonymes en tant que noms ayant pour devoir d'assurer une sorte de paraphrase courte qui conduit à l'anaphore. Leur traduction en turc exige une certaine finalité puisque les synonymes, s'ils peuvent apparaître en une seule unité lexicale simple, sont en même temps capables d'édifier des groupes nominaux efficaces dans le but d'indiquer la ressemblance entre les personnes, les choses ou les faits.

\subsubsection{Anaphore nominale par synonyme}

La traduction d'une anaphore par un synonyme peut se faire par les procédés lexicaux de la langue cible, selon les significations possibles du mot auquel le synonyme se substitue. De ce fait, la synonymie est un vaste champ, et un bon choix dans la traduction des synonymes dépend du registre langagier source aussi bien que de la signification contextuelle de l'élément antérieur auquel renvoie une anaphore synonymique.

23) Nous autres, nous avons tous plus ou moins des maitresses qui nous rendent fous, c'est-à-dire braves. Quand on est amoureux comme un tigre, c'est bien le moins qu'on se batte comme un lion. C'est une façon de nous venger des traits que nous font mesdames nos grisettes.

(Hugo 1862: 663)

a) Bizim tek tük de olsa bizi çıldırtan, yani cesaretimizi artıran sevgililerimiz var. Kaplan gibi bir aşıksan, aslan gibi savaşırsın. O hoppa kızların bize yaptıklarının intikamını böyle alıyoruz.

[Nous, plus ou moins, nous rendant fous avons, c'est-à-dire notre courage augmentant des amantes Le tigre comme amoureux si tu es, le lion comme (tu) fais la guerre. Ces légères filles nous de ce qu'elles font la vengeance ainsi (nous) prenons]

(Hugo 1862/2017: 561, traduit par Yalçıntoklu) 
b) Bize gelince, bizi çıldırtan, yani yüreklendiren sevgililerimiz var. İnsan aşık olursa, en azından aslan gibi vuruşur. O küçük sevgililerimizin bize attıkları okların öcünü almanın bir yoludur bu.

[Nous quant à, nous rendant fou, c'est-à-dire encourageant nos amantes il y a. La personne amoureux si (elle) tombe, au moins, comme le lion se bat. Ces petites de amantes nous qui ont jeté des flèches la vengeance de prendre un moyen c'est ça]

(Hugo 1862/2015: 447, traduit par Karakaya)

c) Nos traductions:

i. Neredeyse hepimiz, bizi çıldırtan, bize kahramanlıklar yaptırtan sevgililere sahibiz. Kaplanlar gibi böylesine aşıkken, aslanlar gibi de vuruşuyoruz. Kadınlarımızın bize attıkları oklardan ${ }^{10}$ öcümüzü almanın bir yoludur bu. [Plus ou moins nous tous, nous rendant fous, nous rendant courageux des amantes nous avons. Des tigres comme ça étant amoureux, des lions comme (on) se bat. De nos femmes à nous, qu'elles jettent des flèches de se venger un moyen c'est]

ii. Neredeyse hepimiz, bizi çıldırtan, bize kahramanlıklar yaptırtan sevgililere sahibiz. Kaplanlar gibi böylesine aşıkken, aslanlar gibi de vuruşuyoruz. Hanımefendilerin, yavuklularımızın bize yaptıklarının acısını çıkarmak gibi bir şey bu.

[Plus ou moins nous tous nous rendant fous, nous rendant courageux des amantes (nous) avons. Des tigres comme ça étant amoureux des lions comme (on) se bat. De nos dames, de nos fiancées, de ce qu'(elles) font, se venger comme une chose cela]

En turc, les proverbes, les anaphores, les reprises et les locutions fonctionnent comme les renforcements naturels de la langue. Ils se trouvent abondamment dans les œuvres littéraires. Akyalçın et Gürcü (2017: 55-68), à la recherche des locutions, proverbes et anaphores dans la littérature turque, confirment que «ceux-ci occupent une place importante dans le trésor linguistique du turc et que l'existence de ces groupes de mots date des premiers temps de l'histoire de la langue turque pour fortifier la langue, la rendre fluide, souple et profonde ${ }^{11} »$.

Dans la première proposition de l'exemple 23, le nom maîtresses a une double fonction syntaxique sur le plan de l'analyse logique: complément d'objet direct du verbe avons et sujet réel du verbe rendent. Il est repris dans la $3^{\mathrm{e}}$ phrase par le procédé de synonymie, sous forme d'un autre groupe nominal mesdames nos grisettes. En turc, parmi d'autres traductions possibles, dans la traduction de Yalçıntoklu, le nom maîtresses est traduit par sevgililerimiz, qui a pour fonction syntaxique le rôle de complément d'objet direct, mais également de sujet de la proposition relative. Donc, dans les langues source et cible, les structures syntaxiques utilisées se ressemblent; voire, elles sont presque identiques. C'est pourquoi, dans la traduction de l'exemple 23 , le procédé anaphorique fonctionne sans problème comme dans la langue source. Le choix d'utiliser un synonyme en vue de procurer l'effet anaphorique source est facilement transposé dans la langue cible. Le terme sevgililerimiz en turc est conforme à ses équivalents contextuels kadınlarımız dans 23ci, hanımefendilerin, yavuklularımızın dans 23cii, o hoppa kızların dans la traduction de Yalçıntoklu et o küçük sevgililerimizin dans la traduction de Karakaya. Ces anaphores cibles recourent à l'équivalence synonymique pour pouvoir refléter au maximum le contenu sémantique, la connotation ironique et le registre familier du groupe nominal mes- 
dames nos grisettes, expression familière, donc pragmatique. Ici, c'est justement le cas de la traduction d'un mot vieilli en langue source.

Comme le conseille Hiernard (2003: 19), « on se pose alors la question suivante: étant donné la phrase où se trouve le terme qui me pose problème, le registre du texte, à quoi puis-je m'attendre ici?» Donc, en considérant «grisette» comme un culturème de l'époque, le procédé d'équivalence utilisé pour la traduction vers le turc implique, pour emprunter les termes d'Andújar Moreno (2013: 73), «le recours au culturème le plus proche de la langue cible; une dénomination qui, même si elle n'est pas exacte dans la plupart des micro segments, offre l'avantage de la familiarité pour le destinataire». C'est grâce à cette familiarité que les termes sources et cibles, porteurs de sens dans les textes de départ et d'arrivée, se lient par formulations culturelles se filtrant facilement d'une culture à l'autre.

De fait, réduire la langue à son strict plan grammatical est une impossibilité pratique et ontologique. Ce qu'il faut travailler, c'est donc le rapport entre les unités inférieures (le lexème, la structure syntaxique, l'énoncé) et leur horizon de sens (le texte et la culture dont ils procèdent sur le plan stylistique, symbolique) en tant qu'un énonciateur singulier s'y exprime, à la fois dans l'intentionnalité de son discours et dans l'inconscient de sa langue. (Szlamowicz: 2011, paragraphe 3)

Prenant cela en considération, Korkut (2008: 154) affirme que «du fait qu'il s'agit en premier lieu des effets de la langue sur les coénonciateurs, nous dirons même que la pragmatique se substitue en quelque sorte à la rhétorique traditionnelle». D'ailleurs, dans une vision de la transférabilité de la transparence des mots, ce qui est autant valable pour l'acte de traduire est que:

on sait que les caractéristiques pragmatiques des textes imposent des contraintes aux traducteurs comme aux lecteurs. Dans certains genres ou domaines, la fidélité à la nuance du sens des notions oblige les traducteurs soit à faire des emprunts soit à créer des néologismes ${ }^{12}$.

On remarque donc que le procédé de synonymie, tout en cherchant des solutions terminologiques communicationnelles qui soient fonctionnelles, assure dans le texte source et cible l'enchaînement de l'idée énoncée. La stratégie sémantique suivie, l'anaphore synonymique, imite le style de la langue source, mais sans engendrer une littéralité erronée ou sans dévider le sens, parce que les deux langues possèdent, dans leur propre système linguistico-culturel, des potentialités analogues d'assortiment de registres, de multitudes de mots synonymes et de séries d'annotations et de connotations possibles. En fait, «le supermème équivalence est la pièce centrale de toute la traduction : il faut le reconnaître, cette notion d'équivalence se montre plus ou moins élastique, ce qui permet d'en déterminer plusieurs formes [...]» (Clas 2003: 608).

Dans la même optique, en expliquant le point de vue de Schleiermacher, Berman et Berner (1999: 15) suggèrent qu'« on ne traduit que des discours, c'est-à-dire de la pensée: celui qui parle ou écrit doit apporter quelque chose à la langue, ce qui interdit la simple répétition ».

C'est pourquoi dans notre segment (4), l'anaphore synonymique, qui est une anaphore nominale, s'emploie de façon beaucoup plus créative et féconde par rapport aux anaphores pronominales, à condition de prendre strictement en compte les équivalences terminologiques et les registres langagiers. D’ailleurs la typologie des schémas d'équivalence de Ballard soutient la même idée: 
l'équivalence directe aura un effet de simple substitution entre des termes et des structures qui semblent se correspondre et l'équivalence indirecte offrira des différences de diverses natures (autres que la graphie) par rapport à la base. (Ballard 2006: 77-86)

À propos, en tant que mode de traduction, l'interprétation de ce genre d'anaphore devra bien correspondre aux exigences sémantico-contextuelles comme dans le segment (4). Comme le désigne déjà Lederer (1994: 51), "l'équivalence est une correspondance inédite». Autrement dit, elle réapparaît et se renouvelle. À cet égard, la découverte des équivalences pour les anaphores synonymiques cibles dépendra incontestablement de la créativité du traducteur-auteur en fonction de sa connaissance de la langue et de la culture cibles, ainsi que de celle de la langue source dans une perspective particulière, principalement contextuelle et notamment pragmatique. Du reste, Blanchet (1995: 85) affirme que, d'après Austin, «la signification est la réunion du sens et de la référence, le sens pouvant avoir un effet pragmatique».

Les segments suivants possèdent les mêmes incidences classiques concernant l'anaphore par synonyme dans les langues source et cible.

24) Qu'elle est jolie! Qu'elle est jolie. C'est un Greuze. Tu vas donc avoir cela pour toi seul, polisson! Ah! mon coquin, tu l'échappes belle avec moi [...].

(Hugo 1862: 734)

a) Ne kadar güzel! Ne kadar güzel! Greuze'ün tablosundan çıkmış gibi. Hayta, demek onu kendine ayırdın! Ah! Çapkın,[...].

[Comme joli! Comme joli! De Greuze de son tableau sortie comme. Vagabond, c'est que la pour toi-même (tu) a prise! Oh! Don Juan [...]].

(Hugo 1862/2017: 706, traduit par Yalçıntoklu)

b) Aman ne kadar güzel kız! Ne güzel! Greuze'ün bir tablosu! Çapkın! Ah! seni yaramaz seni!

[Quelle jolie fille! Comme jolie! De Greuze un tableau! Don Juan! Oh! toi polisson!]

(Hugo 1862/2015: 577, traduit par Karakaya)

c) Ne kadar da güzel! Greuze tablosu gibi adeta. Onu yalnız kendine saklıyorsun, çapkın seni...Şu serseriye bak hele [...].

[Si jolie! Greuze de un tableau. La seulement pour toi (tu) caches, Don Juan toi... Ce coquin regardez-moi ...]

(Notre traduction)

Dans notre traduction, serseri (mon coquin) est la reprise synonymique de çapkın (polisson). Dans la traduction de Yalçıntoklu, çapkın est le reflet synonymique de hayta. Dans la traduction de Karakaya, seni yaramaz seni symbolise çapkın. Ainsi, la traduction des références auxquelles s'attachent les anaphores et celle de leur(s) reprise(s) synonymique(s) se reproduisent pareillement au style source.

Émanant de la littérature turque, les segments ci-après affirment de façon complémentaire l'usage des synonymes en tant qu'anaphores en turc:

25) Kerim kimsiz kimsesiz, anasız babasız, kardeşsiz büyümüş [...].

[Kerim orphelin, sans mère sans père, sans frère sans sœur a grandi [...]]

(Kemal 2012: 18)

a) Kerim avait grandi orphelin, sans mère ni père, ni frère ni sœur [...].

(Notre traduction) 
26) "Unutmaz" dedi Kerim, " o unutsa bile onun adamları unutmaz. Hane halkı, çocukları unutmaz".

[(Ils ) n'oublierait pas dit Kerim. «lui s'(il) oublie même ses amis n'oublieront pas. La maison de les membres les enfants n'oublient pas]

(Kemal 2012: 21)

a) «Il ne les oublierait pas» dit Kerim. "Même si, lui, il les oublie, ses proches, ses supporteurs ne les oublieraient pas». La famille n'oublie jamais les enfants.

(Notre traduction)

27) Dünyanın ortasında yapayalnız, tek başlarına kalmışlardı.

[Du monde au milieu tout seul, esseulés, (ils) étaient restés]

(Kemal 2012: 22)

a) Ils étaient restés tout seul sur la planète, esseulés, sans compagnie.

(Notre traduction)

Dans l'exemple 25, se rattachant au sujet réel, la propriété citée kimsesiz (orphelin) est répétée par le biais de son synonyme anasız babasız (sans mère ni père). Dans l'exemple 24, le sujet onun adamlar1 (ses proches/ses supporteurs) est anaphorisé par son synonyme hane halkı (sa famille). Enfin, dans l'exemple 27, la description qualificative yapayalnız (tout seuls) est redite telle que tek başlarına (esseulés, sans compagnie).

L'anaphore nominale constituée par une périphrase en propose un autre exemple.

\subsubsection{Anaphore nominale par périphrase}

Les anaphores nominales qui utilisent une périphrase fonctionnent dans le même sens que les anaphores nominales synonymiques. Relative à la forme stylistique employée, la périphrase est une figure de style qui embellit le discursif, orne les paroles de l'auteur et remet le texte dans un élan plus frappant aux yeux du destinataire. Sans exprimer davantage, elle explique et développe l'histoire, bien qu'elle n'y ajoute aucune information supplémentaire.

Cette façon détournée d'exprimer les choses dans le but de reprendre le propos présenté se traduit en turc par un procédé semblable. Toutefois, dans les deux langues, la périphrase a une plus vaste capacité de créer une anaphore que les procédés cités plus haut. Elle s'expose de manière beaucoup plus ample que le synonyme. Pourtant, pour le traducteur qui va et vient entre les textes source et cible, elle peut causer un éventuel glissement de sens, car elle possède une nature largement ouverte à l'interprétation comme dans le segment (6). Dans ce sens, «la traduction-analyse s'impose au traducteur pour contrôler sa pulsion traductrice» (Guidère 2010: 2).

28) Aujourd'hui, et depuis vingt ans, l'embarcadère du chemin de fer d'Orléans est là, à côté du vieux faubourg, et le travaille. Partout où l'on place, sur la lisière d'une capitale, l'embarcadère d'un chemin de fer, c'est la mort d'un faubourg et la naissance d'une ville. Il semble qu'autour de ces grands centres du mouvement des peuples, au roulement de ces puissantes machines, au souffle de ces monstrueux chevaux de la civilisation qui mangent du charbon et vomissent du feu, la terre pleine de germes tremble et s'ouvre pour engloutir les anciennes demeures des hommes et laisser sortir les nouvelles.

(Hugo 1862: 241)

a) Bugün ve yirmi yıldan beri Orléans garı eski mahalleyi huzursuz eder. Bir başkentin kıyısına inşa edilen bir gar, kenar mahallenin ölümü, bir kentin doğumu 
anlamına gelir. İnsan hareketliliğinin yoğunlaştığı bu büyük merkezlerinin etrafında, bu güçlü trenlerin uğultusunun, uygarlığın kömür yiyip ateş kusan bu korkunç atlarının soluğunun ortasında, tohumlarla dolu toprak, adeta titreyerek açılır, insanların oturdukları eski evleri yutar ve yerlerine yenilerinin yükselmesini sağlar.

[Aujourd'hui et vingt ans depuis Orléans de la gare vieux quartier rend mal à l'aise. Une capitale au bord construit une gare, un faubourg de la mort, une ville de la naissance signifie. De la foule des hommes ou s'intensifie ces grands de centres autour, ces forts de trains le bruit, de la civilisation du charbon mangeant du feu vomissant ces terribles chevaux l'haleine au sein de, avec des grains pleine la terre, comme frémissant, s'ouvre, les hommes où habitent vieilles des maisons englouti et à leur place des nouvelles la construction procure]

(Hugo 1862/2017: 513, traduit par Yalçıntoklu)

b) Bugün, yirmi yıl var ki Orléans demiryolunun istasyonu orada, eski mahallenin yanıbaşındadır ve onu için için işleyip değiştirmektedir. Nerede bir başketin sınırı üstüne bir demiryolu istasyonu konursa, bu bir mahallenin ölümü ve bir şehrin doğuşu olur. Sanki, halkların bu büyük hareket merkezlerinin çevresinde bu güçlü makinelerin işlemesiyle, kömür yiyip ateş kusan bu canavar gibi uygarlık beygirlerinin soluğuyla, tohum dolu toprak titreyip açllır ve insanların eski barınaklarını yutarak yerine yenilerini çıkarır.

[Aujourd'hui vingt ans il y a que Orléans de le chemin de fer de la station là-bas, le vieux quartier tout près de est et le profondément travaille et modifie. Là où d'une capitale la frontière sur un chemin de fer une station de se met, cela d'un quartier la mort et d'une ville la naissance est. Comme si, les peuples de ces grands agités centres autour, les fortes machines avec le fonctionnement, du charbon mangeant du feu vomissant ce monstre comme la civilisation des chevaux par l'haleine, de grains pleine de la terre frémissante s'ouvre et des hommes vieillis leurs abris en engloutissant à leur place les nouveaux sorte]

(Hugo 1862/2015: 514, traduit par Karakaya)

c) Nos traductions:

i. Bugün, yirmi yıldan beri, Orléans demiryolu istasyonu orada, şehrin kıyısındaki eski mahallenin yanı başındadır ve burasını işleyip değiştirmektedir. Bir başkentin sınırlarında bir demiryolu istasyonu kurulduğu zaman, bu, bir kenar mahallenin ölümü, bir kentin doğuşu demektir. Sanki, bu güçlü makinelerin gümbürtüleriyle, uygarlığın ateş kusan, kömür yiyen canavar beygirlerininin nefesleriyle dolu, hareketli ve kalabalık bu büyük merkezlerin çevresindeki, tohumları filizlenen toprak titrer, açılır ve insanların eski evlerini yutarak yenilerini ortaya çıkarır.

Aujourd'hui, vingt ans depuis, Orléans chemin de fer de la station là-bas, de la ville au bord du vieux quartier tout près est et le travaille et modifie. D'une capitale aux frontières chemin de fer de une station, c'est établi quand, cela, d'un faubourg la mort, d'une vile la naissance signifie. Comme si, de ces puissantes machines avec le bruit, de la civilisation du feu vomissant du charbon mangeant, des monstrueux chevaux avec les haleines pleins, agités et peuplés de ces grands centres autour, dont les grains germaient la terre frémissait, s'ouvrait et des gens vieilles les maisons en engloutissant les nouvelles en sortait [Glose]

(Notre traduction)

ii. Bugün, yirmi yıldan beri, Orléans demiryolu istasyonu orada, şehrin kıyısındaki eski mahallenin yanı başındadır ve burasını işleyip değiştirmektedir. Bir başkentin sınırlarında bir demiryolu istasyonu kurulduğu zaman, bu, bir 
kenar mahallenin ölümü, bir kentin doğuşu demektir. Bu kocaman yerleşim bölgelerinin çepeçevresinde sağır eden gürültüsüyle, kömürle beslenip çal1şan, etrafa kapkara dumanlar saçan bu çağdaş ulaşım araçlarının güçlü lokomotiflerinin, üzerinden geçtikleri verimli topraklar adeta yarılırcasına sarsılır; mahalleler gelişir, insanların oturdukları eski evlerin yerine de yenileri inşa edilir.

[Aujourd'hui, vingt ans depuis, Orléans chemin de fer de la station là-bas, de la ville au bord du vieux quartier tout près est et le travaille et modifie. D'une capitale aux frontières chemin de fer de une station, c'est établi quand, cela, d'un faubourg la mort, d'une ville la naissance signifie. De ces immenses résidentielles régions autour, assourdissant avec le bruit, se nourrissant avec du charbon et fonctionnant, aux alentours tout noir des fumées répandant ces modernes de transport moyens puissantes des locomotives, sur lesquelles fertiles les terres (elles) passaient comme si elles éclataient secouaient; des quartiers se développent; les gens que habitent vieilles des maisons à la place les nouvelles se construisent]

(Nos traductions)

Dans l'exemple 28, l'embarcadère de chemin de fer qui désigne la station ou la gare de train (implicitement les trains, les locomotives et les wagons dans leur ensemble), est périphrasé d'abord dans la troisième phrase par ces grands centres $d u$ mouvement des peuples, puis par ces puissantes machines, au souffle de ces monstrueux chevaux de la civilisation qui mangent du charbon et vomissent $d u$ feu.

Ces trois anaphores nominales embrassent donc trois périphrases, soutenues trois fois par le même adjectif démonstratif ces. La dénotation et l'embarcadère de chemin de fer (demiryolu istasyonu), est périphrasée en turc avec premièrement bu hareketli ve kalabalık büyük merkezler (ces grands centres du mouvement des peuples) dans notre traduction, deuxièmement, par bu güçlü makineler (ces puissantes machines), dans la traduction de Karakaya, et, troisièmement, $(3 / 5 \mathrm{c})$ par uygarlığın ateș kusan, kömür yiyen bu canavar beygirleri (ces monstrueux chevaux de la civilisation qui mangent du charbon et vomissent $d u$ feu), dans la traduction de Yalçıntoklu. Deux périphrases traduites en turc contiennent, comme dans la langue source, l'adjectif démonstratif bu (ces), qui renforce et identifie les périphrases de sens abstrait en tant que sujet concret. Très proches des nôtres, les traductions de Yalçıntoklu et Karakaya se veulent incontestablement similaires.

En ce qui concerne la traduction, le traducteur joue un rôle important pour faire remarquer au destinataire qu'il s'agit des trains de l'époque, tout en dessinant leur description métaphorique qui forme l'anaphore. Celle-ci est donc associative. Sans citer explicitement les trains, les locomotives, les wagons, les fumées, les voyageurs et les travailleurs, l'auteur y fait allusion. Le traducteur est donc responsable de recréer en turc la même relation métaphorique entre l'anaphore et son antécédent, sans jamais nuire à l'illustration de la représentation métaphorique ni à son intensité, et en transmettant la même valeur sémantique. Dans ce but, le moyen utilisé dans la traduction de l'exemple 28 est toujours l'anaphore nominale par périphrase, qui reste presque totalement fidèle à la langue source. Ainsi, l'anaphore nominale périphrastique ne contrarie pas le discursif du turc ni sur le plan grammatico-structural et syntaxique ni au niveau lexical. Elle est totalement applicable dans la langue cible et digne de créer l'effet désiré par l'auteur du texte source. 
En somme, il convient de souligner que, du point de vue du style, la traduction qui laisse intacte l'anaphore nominale et faite par l'entremise d'une périphrase source, diffère de la périphrase faite par le traducteur en fonction de sa décision concernant sa traduction. Dans le cas de la fidélité à la périphrase source, le traducteur suit volontairement le principe stylistique de l'auteur (parce que le turc le permet bien ici) tout en reproduisant la même anaphore métaphorique cible. En revanche, dans le cas de l'utilisation de la périphrase nominale pour raison de nécessité traductologique qui provient des décisions stratégiques du traducteur, la périphrase n'est plus un reflet identique de la langue source. Là, elle est plutôt une sorte de stratégie d'explicitation métaphorique qui figure entre autres dans l'activité rédactionnelle et qui dépend des pertinences proposées par l'outillage interlinguistique. Dans ce sens, l'anaphore nominale reliée à une périphrase interpelle l'anaphore conceptuelle telle que ces monstrueux chevaux de la civilisation (uygarlığın canavar beygirleriu), dans laquelle l'auteur développe son point de vue, et le traducteur émet exactement ce point de vue. Cependant, le traducteur peut adopter une approche beaucoup plus libre.

Cette approche est configuratrice et lorsque la configuration en question est faite, cette situation prouve qu'en turc cible, la traduction de l'anaphore nominale en périphrase originale peut se rapprocher facilement de la langue source, parce que même si c'est une métaphore qui remplace le sujet concret, la visée est de transmettre la pensée critique originale de l'auteur. On arrive donc ici à une double pratique d'expression possible: soit le descriptif métaphorique concret prime grâce à l'abstraction source pour une traduction littérale conceptuelle du français vers le turc, comme dans notre traduction; soit une dénotation explicitée authentique transmet l'anaphore périphrastique et effectue le même rôle, comme dans les traductions de Yalçıntoklu et Karakaya. Dans ce contexte, l'important pour le traducteur est de pouvoir comprendre et de faire comprendre au destinataire la pensée de l'auteur. Comme l'exprime Schleiermacher,

[p]artout où le discours n'est pas totalement lié à des objets visibles ou à des faits extérieurs qu'il suffit d'énoncer, partout où celui qui parle pense de manière plus ou moins indépendante, veut par conséquent s'exprimer, il se trouve vis-à-vis de la langue dans un rapport double et son discours n'est correctement compris que dans la mesure où ce rapport l'est aussi. (Schleiermacher 1813/1999: 41)

Dans nos traductions ces grands centres du mouvement des peuples et de ces puissantes machines, nous avons transporté l'idée vers la langue cible en établissant la relation entre les mots et l'idée. Pourtant, les mots sont bien susceptibles d'être transformés tandis que l'idée reste la même. Alors, dans le cas de la traduction directe de l'implicite situé dans l'anaphore périphrastique, comme ces grands centres $d u$ mouvement des peuples, le traducteur est libre de transmettre le style lexical source. Ou alors, il choisit de transporter l'anaphore périphrastique en explicitant les objets et les réalités physiques connotés par l'anaphore périphrastique dont la stylistique est neutralisée. Pour ainsi dire, manger du charbon et vomir du feu serait en turc consommer le combustible de l'époque et en conséquence polluer l'environnement par une fumée empoisonnante, insalubre, malfaisante, comme explicité de façon approximative tel que sağır eden gürültüsüyle, kömürle beslenip çalıșan, etrafa kapkara dumanlar saçan. D’ailleurs, Konmuş (2007: 25) atteste qu'«il est très rare en turc, de 
retrouver des phrases composées inversées, syntaxiquement sophistiquées. Les notions abstraites sont exprimées en mots concrets ${ }^{13}$ ".

Si l'on croit Ladmiral (1979/1994: 219), «c'est ainsi que le traducteur tend à être souvent placé devant l'obligation de "paroliser» des éléments de langue source [...] ». Il s'agit surtout dans la traduction de la périphrase, d'un genre de pacte négocié dans la conscience du traducteur. Une fois l'engagement instauré, les deux textes, texte source qui se qualifie de boussole et texte cible qui s'oriente à diverses reprises d'après le champ magnétique, s'hypothèquent de part et d'autre, ce qui permet la naissance de la création textuelle cible. Sous ce rapport, Eco (2003: 37) souligne que «la traduction, et c'est évidemment un principe en traductologie, n'est pas entre systèmes, mais entre textes ${ }^{14}$.»

Cela dépendra quand même du genre textuel et de l'idéologie de la traduction. Les exemples de traductions probables présentés ci-dessous en justifient l'évidence.

29) Un président assisté d'un jardinier, un bonhomme qui croyait continuer Lamoignon et un autre bonhomme qui croyait continuer Lenôtre, l'avaient contourné, taillé, chiffonné, attifé, façonné pour la galanterie [...].

(Hugo 1862: 485)

a) Bir mahkeme başkanıyla yardımcısı bir bahçıvan, yani Lamoignon'un*15 geleneğini sürdürdüğüne inanan bir adamla Lenôtre'un ${ }^{\star * 16}$ geleneğini sürdürdügüne inanan başka bir adam, bu bahçeyi çerçevelemişler, kesmişler, biçmişler, cicili bicili süslemişler, gönül maceralarına uygun bir biçime sokmuşlardı.

[Un tribunal le président de et son assistant et un jardinier c'est-à-dire de Lamoignon, la tradition qu'il continue qui croit un homme, Lenôtre, la tradition qu'il continue qui croit autre un homme, ce jardin avaient entouré, coupé, modelé, méticuleusement orné, d'amour aux aventures convenable d'une façon avaient transformé]

(Hugo 1862/2000: 117, traduit par Atayman)

b) Lamoignon'un izinden gittiğini sanan bir başsavcı ve Lenôtre'un izinden gittiğini sanan bir bahçıvan çapkınlık adına onun şeklini bozmuş, her yanını kesip biçmiş, çirkineştirmişti (...).

Lamoignon suivre qui croyait un procureur général et Lenôtre suivre qui croyait un jardinier des coquineries au nom de sa forme avait défigurait, partout avait découpé, modelé et avait enlaidi]

(Hugo 1862/2017: 187, traduit par Yalçıntoklu)

c) Bir başkanla bir bahçıvan, yani Başsavcı Lenôtre'un takipçisi olduğuna inanan bir mahkeme başkanı ile ona yardımcı olan ve bahçe tasarımcısı Lamoignon'un tarzını benimsediğine inanan bir bahçıvan, bahçeyi çepeçevre şekillendirmişler, kesmiş biçmiş, zevksizce allamış pullamış, çapkınlığa uygun hale getirmişlerdi [...].

[Avec un président un jardinier, c'est-à-dire le procureur général de Lenôtre successeur être qui croyait un tribunal président de et qui l'assiste et le concepteur de jardin Lamoignon le style de adopter qui croyait un jardinier, le jardin tout autour avaient façonné, découpé et modelé, sans goût, de façon exagérée avaient orné à la coquinerie propice avaient rendu]

(Notre traduction)

Dans l'exemple 29, Hugo fait appel à deux personnages historiques connus. Le premier est un juge français qui s'appelle Lamoignon, le deuxième est un jardinier 
français qui s'appelle Lenôtre. Dans les traductions vers le turc, prononcer lesdits noms propres sans en donner une explication ou une annotation mettrait le lecteur turc dans une sorte de gêne d'interprétation. De cette gêne naîtrait un malaise de compréhension: "qui sont ces personnages?», «dans quel but l'auteur les cite-t-il?», «est-ce qu'ils ont déjà été mentionnés dans les pages précédentes du roman?». La périphrase offre une formule traductionnelle soit sous forme d'une apposition comme dans notre traduction, soit sous forme d'annotation utilisée par le traducteur Atayman, soit en paraphrasant directement le président par bir bașsavc1 [procureur général] tel que dans la traduction de Yalçıntoklu. Dans ce dernier cas, l'anaphore source est engloutie par l'alliance du nom propre avec la profession d'où le turc cible incise l'anaphore.

De semblables procédures de périphrases enrichissent les textes littéraires en turc, en établissant des stratégies de répétitions, comme dans les deux exemples qui suivent:

30) Kerim’i de öldürürler benim yüzümden. $\mathrm{O}$ da, bu yaşında kara topraklara gider. [Kerim aussi (ils) tueraient moi à cause de. Lui et, cet à âge, noires sous les terres se rendrait]

(Kemal 2012: 23)

a) Ils tueraient Kerim aussi, à cause de moi. Et lui, à cet âge-là, il serait mis sous terre.

(Notre traduction)

31) Kerim de söylüyor bu türküleri. Kerim de Kafkasya diyor başka bir şey demiyor. En güzel türküleri o söylüyor. [...] Kafkasya diyordu, altın kaplı, kayalıklarından çiçekler fışkıran, bize yasaklı dağlarımız, diyordu.

[Kerim aussi dit ces airs. Kerim aussi le Caucasse dit d'autre rien ne dit. Les plus beaux airs il chante. [...] Le Caucasse (il) disait, d'or couvert, de ses rochers les fleurs jaillissant, à nous interdites nos montagnes]

(Kemal 2012: 24)

a) Kerim aussi, il chante ces airs. Il ne prononce que le Caucase, rien que le Caucase. Il chante les plus beaux airs. Il chante le Caucase, nos montagnes qui nous sont interdites d'où jaillissent des fleurs dans les rochers, le Caucase aux portes d'or, disait-il.

(Notre traduction)

Dans l'exemple 30, le verbe source öldürürler (tueraient) est périphrasé en kara topraklara gider (serait mis sous terre). Dans l'exemple 30, le complément d'objet direct source Kafkasya (Caucase), signifiant une chaîne de montagnes, est décrit en détail par la périphrase nominale altın kaplı, kayalıklarından çiçekler fışkıran, bize yasaklı dağlarımız. La périphrase anaphorique source dessine, détaille et clarifie ainsi son antécédent.

\section{Résultats}

En vue d'examiner la correspondance anaphorique pronominale et nominale du couple de langues français-turc, différentes anaphores ont été étudiées dans divers segments. Nous avons constaté que, pour les anaphores pronominales sources, la traduction recourt le plus à l'omission, rarement à la littéralité, parfois à la transpo- 
sition. Pour les anaphores nominales, la traduction se sert habituellement de la littéralité, généralement de l'équivalence, peu fréquemment de la transposition.

En conséquence, les anaphores pronominales sources, assurées notamment par des pronoms personnels et pronoms personnels compléments, sont en grande partie omises. Il arrive quand même qu'elles soient traduites littéralement. En outre, il se peut que les anaphores assurées par des pronoms démonstratifs composés soient omises ou transposées. Contrairement aux pronoms anaphoriques, les anaphores nominales sont beaucoup plus aptes à ne pas être omises. Voire, la plupart du temps, on ne peut pas passer sous silence leur caractère explicatif, notamment lorsqu'elles jouent le rôle de sujet réel au niveau de l'axe syntaxique. On distingue bien que pour les langues source et cible, la périphrase peut faire connaître et dessiner le contenu d'un évènement ou d'une pensée sous forme d'anaphore nominale, sans poser de problème ou sans créer une ambiguïté traductologique. Elles sont majoritairement traduites littéralement. Les anaphores nominales assurées par les périphrases peuvent être traduites littéralement ou par équivalence. Celles-ci, assurées par des synonymes d'origine culturelle, se traduisent par équivalence. En revanche, l'anaphore nominale produite par le terme générique est susceptible d'être traduite littéralement ou par transposition. Dans le cadre de cette étude, les résultats se limitent auxdits segments étudiés.

Les tableaux ci-dessous résument la correspondance anaphorique des cinq exemples sources et cibles sujets de notre étude.

TABLEAU 1

Correspondance traductionnelle des anaphores pronominales français-turc

\begin{tabular}{|c|c|c|c|c|c|c|c|c|}
\hline \multirow{2}{*}{\multicolumn{2}{|c|}{ Segment }} & \multirow{4}{*}{$\begin{array}{l}\text { Anaphore } \\
\text { pronominale source }\end{array}$} & \multirow{3}{*}{$\begin{array}{l}\text { Anaphore } \\
\text { pronominale } \\
\text { cible }\end{array}$} & \multicolumn{4}{|c|}{$\begin{array}{l}\text { Procédés } \\
\text { traductologiques }\end{array}$} & \multirow{3}{*}{$\begin{array}{l}\text { Cause du choix } \\
\text { traductionnel } \\
\\
\text { Inutilité sémantique. } \\
\text { Effet peu naturel }\end{array}$} \\
\hline & & & & 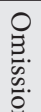 & 点. & 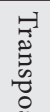 & 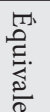 & \\
\hline \multirow{2}{*}{1} & \multirow{2}{*}{$1 / 1$} & & & $\mathrm{X}$ & & & & \\
\hline & & & ondan & & $\mathrm{X}$ & & & $\begin{array}{l}\text { Nécessité anatomique } \\
\text { syntaxico-sémantique }\end{array}$ \\
\hline \multirow{3}{*}{1} & \multirow{3}{*}{$2 / 1$} & $\begin{array}{l}\text { pronom personnel } \\
(e l l e)\end{array}$ & o & $\mathrm{X}$ & & & & Inutilité syntaxique \\
\hline & & \multirow{2}{*}{$\begin{array}{l}\text { pronom démonstratif } \\
\text { composé }(c e l u i-c i)\end{array}$} & \multirow{2}{*}{ o } & $\mathrm{X}$ & & & & $\begin{array}{l}\text { Inutilité syntaxique. } \\
\text { Effet d'encombrement } \\
\text { lexical }\end{array}$ \\
\hline & & & & & & $\mathrm{X}$ & & Nécessité explicative \\
\hline
\end{tabular}


TABLEAU 2

Correspondance traductionnelle des anaphores nominales français-turc

\begin{tabular}{|c|c|c|c|c|c|c|c|c|}
\hline \multirow{2}{*}{\multicolumn{2}{|c|}{ Segment }} & \multirow{4}{*}{$\begin{array}{l}\text { Anaphore } \\
\text { nominale } \\
\text { source }\end{array}$} & \multirow{3}{*}{$\begin{array}{l}\text { Anaphore } \\
\text { nominale } \\
\text { cible }\end{array}$} & \multicolumn{4}{|c|}{$\begin{array}{l}\text { Procédés } \\
\text { traductologiques }\end{array}$} & \multirow{3}{*}{$\begin{array}{l}\text { Cause du choix } \\
\text { traductionnel } \\
\\
\begin{array}{l}\text { Utilité syntaxico- } \\
\text { sémantique }\end{array}\end{array}$} \\
\hline & & & & \multirow{2}{*}{ 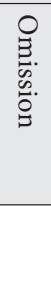 } & \multirow{2}{*}{ 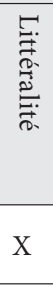 } & \multirow{2}{*}{ 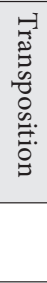 } & \multirow{2}{*}{ 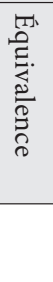 } & \\
\hline \multirow{2}{*}{3} & \multirow{2}{*}{$2 / 3$} & & & & & & & \\
\hline & & & bu olay & & & $\mathrm{X}$ & & $\begin{array}{l}\text { Concrétisation } \\
\text { sémantique }\end{array}$ \\
\hline \multirow[t]{2}{*}{4} & \multirow[t]{2}{*}{$3 / 4$} & \multirow[b]{2}{*}{$\begin{array}{l}\text { synonyme } \\
\text { (mesdames } \\
\text { nos grisettes) }\end{array}$} & $\begin{array}{l}\text { Kadinlar1- } \\
\text { miz }\end{array}$ & & & & $\mathrm{X}$ & $\begin{array}{l}\text { Utilité pragmatico- } \\
\text { culturelle }\end{array}$ \\
\hline & & & $\begin{array}{l}\text { Hanım- } \\
\text { efendiler, } \\
\text { yavuklu- } \\
\text { larımız }\end{array}$ & & & & $\mathrm{X}$ & $\begin{array}{l}\text { Agitation mentale } \\
\text { pragmatico-culturelle }\end{array}$ \\
\hline \multirow[t]{2}{*}{5} & \multirow[t]{2}{*}{$3 / 5 a$} & \multirow{2}{*}{$\begin{array}{l}\text { périphrase } \\
\text { (ces grands } \\
\text { centres du } \\
\text { mouvement } \\
\text { des peuples) }\end{array}$} & $\begin{array}{l}\text { hareketli ve } \\
\text { kalabalık } \\
\text { büyük } \\
\text { merkezler }\end{array}$ & & $\mathrm{X}$ & & & Nécessité descriptive \\
\hline & & & $\begin{array}{l}\text { bu kocaman } \\
\text { yerleşim } \\
\text { bölgeleri }\end{array}$ & & & & $\mathrm{X}$ & $\begin{array}{l}\text { Nécessité de compacité } \\
\text { définitionnelle }\end{array}$ \\
\hline \multirow{2}{*}{5} & \multirow{2}{*}{$3 / 5 b$} & \multirow{2}{*}{$\begin{array}{l}\text { périphrase } \\
\text { (ces } \\
\text { puissantes } \\
\text { machines) }\end{array}$} & $\begin{array}{l}\text { bu güçlü } \\
\text { makineler }\end{array}$ & & $\mathrm{X}$ & & & Utilité sémantique \\
\hline & & & $\begin{array}{l}\text { güçlü } \\
\text { lokomotif-ler }\end{array}$ & & & & $\mathrm{X}$ & Utilité explicative \\
\hline \multirow{2}{*}{5} & \multirow{2}{*}{$3 / 5 c$} & \multirow{2}{*}{$\begin{array}{l}\text { périphrase } \\
\text { (ces } \\
\text { monstrueux } \\
\text { chevaux de la } \\
\text { civilisation) }\end{array}$} & $\begin{array}{l}\text { uygarlığın } \\
\text { canavar } \\
\text { beygirleri }\end{array}$ & & $\mathrm{X}$ & & & Utilité sémantique \\
\hline & & & $\begin{array}{l}\text { bu çağdaş } \\
\text { ulaşım } \\
\text { araçları }\end{array}$ & & & & $\mathrm{X}$ & $\begin{array}{l}\text { Nécessité } \\
\text { d'explicitation }\end{array}$ \\
\hline
\end{tabular}

\section{En guise de conclusion}

La traduction, du français vers le turc, des pronoms personnels, des pronoms personnels compléments et des pronoms démonstratifs anaphoriques est un phénomène délicat. Ces pronoms ne peuvent pas toujours être transposés littéralement en turc. S’ils le sont, le sens peut être changé ou même altéré. De même, leur traduction littérale peut alourdir la traduction et de fait la rendre non idiomatique. En tant que langue agglutinante, le turc dispose largement, pour ses locuteurs et lecteurs, d'une grande connivence qui libère les implicites, les elliptiques et les incises. Cela est un fait ténu qui oblige le français et le turc à se distancier l'un de l'autre dans la perspective de traduction des anaphores pronominales.

En français, les pronoms personnels et démonstratifs en turc, à l'instar de leurs fonctions grammaticales, sont parfaitement capables de générer des anaphores, mais 
il est incontestablement souhaitable que leur emploi répétitif dans une traduction soit bien maîtrisé par le traducteur.

De plus, dans la traduction des anaphores nominales du français vers le turc, à l'exemple d'anaphores procurées par un terme générique, un synonyme et une périphrase, ces types d'anaphores se réconcilient vite au cœur des deux langues.

En définitive, considérant la traduction du français vers le turc, la transposition directe des anaphores dans la langue cible, comme elles le sont dans la langue source, gagne de la vitesse croissante en direction de la nature abstraite du discours, versus celle qui permet la verbalisation concrète. Ce qui signifie que les formes de structure purement grammaticales dont la vocation est de créer des anaphores telles que les anaphores grammaticales sont soumises davantage à l'omission en turc que les formes renvoyant à l'abstrait.

Il est vrai que le phénomène qui consiste à établir des traductions dans la pratique de l'acte traductologique ouvre la voie à un ensemble très étendu de postulats qui doivent être vérifiés pour devenir des produits finaux. En tenant compte de la dissemblance de la structure syntaxique entre le français et le turc, traduire les anaphores pronominales et nominales dans les deux langues demande une attention particulière. Ce trait propre au français et au turc peut être source de traductions surchargées ou lacunaires. C'est la raison pour laquelle le futur traducteur du français, dont la langue maternelle ou de spécialité est le turc, devra sensibiliser sa perception des procédés grammaticaux et stylistiques qui se servent de l'anaphore en français. En conséquence, il développera ses propres stratégies dans la langue turque, pour disposer d'une bonne saisie sémantique au sein de la complicité langue source-langue cible.

Dans cette perspective, la formation de l'apprenant en direction de l'analyse logique syntaxique et de l'analyse du discours aidera le futur traducteur à prendre des décisions quant aux bonnes solutions traductionnelles.

Par ailleurs, pour les apprenants de langues étrangères, en plus de l'enseignement-apprentissage de la langue étrangère, une profonde connaissance de leur langue maternelle et la pratique de celle-ci ne doivent pas non plus être négligées et la formation dans ladite langue a une importance aussi cruciale que celle de la langue étrangère. Il convient de ne pas oublier que les traductions erronées ou qui manquent d'efficience ne reposent pas toujours sur la carence ou l'inattention se rapportant au savoir ou à la pratique de la langue étrangère. Parfois, leur provenance consiste en la saisie insuffisante ou en des savoirs boiteux dans la langue maternelle. Il vaut mieux ne pas oublier qu'il est tout à fait possible de traduire une langue vers cette même langue. En fait, cela paraît être la toute première étape de l'acte de traduire une langue vers une autre.

\section{NOTES}

1. Il s'agit de l'Université Marmara (Turquie), section de traductologie du français vers le turc où l'auteur a déjà enseigné et de l'Université Adnan Menderes/Turquie, section de langue et littérature françaises où l'auteur continue d'enseigner aux étudiants en licence 1, 2, 3 et 4.

2. Hugo, Victor (1862). Les Misérables. Paris: J. Hetzel et A. Lacroix. Bibliothèque Nationale de France. BNF Gallica. www.data.bnf.fr/13316296/victor_hugo_les_misérables/. 798 p.

3. Parmi les premières traductions d'ouvrages littéraires effectuées vers le turc, Les Misérables sont la deuxième œuvre romanesque qui est traduite en turc par Münif Paşa en 1862 et publiée dans le journal Ruzname-i Ceride-i Havadis. Le roman a été traduit encore en 1880 sous le titre de Sefiller par Şemsettin Sami Bey, puis une troisième fois en 1934 par Hasan Bedrettin Bey. 
4. Kemal, Yaşar (2012). Çıplak ada, Çıplak Deniz. Bir Ada Hikayesi 4. Istanbul: Yapı Kredi Yayınları..

5. À part les traductions que nous-mêmes avons faites, nous nous sommes servies des traductions publiées de Volkan Yalçıntoklu (2017). Sefiller I et II. İstanbul: Türkiye ş Bankası Yayınları. ISBN 978-605-332-474-4 ; Cenap Karakaya (2015). Sefiller I et II. İstanbul: İletişim Yayınları. ISBN -13:978-975-05-1155-4 ; Nurten Genç (2004). Sefiller I et II. İstanbul: Oda Yayınları. ISBN 975385-334-3 et ISBN 975-385-335-1; Mete Aydoğan (2003). Sefiller I et II. İstanbul: Mavi Yelken Yayınları. ISBN 9799756684121; Semih Atayman (2005). Sefiller I ve II. İstanbul: Bordo Siyah Yayınları. ISBN 975-8688-55-3.

6. Traduction vers le français du titre du roman: «La mer nue, l'île nue. Une histoire d'île 4». 2012. Istanbul: Yapı Kredi Yayınları.

7. Texte original en turc de la citation traduite: «Türkçe'deki kişi zamirlerinin kullanımı Batı dillerinden oldukça farklıdır. Eklemeli bir dil olduğu için, kişi bilgisi hem yüklemdeki çekim ekleriyle hem de bağımsız zamirlerle verilebilmektedir. Bu nedenle Türkçe "zamir düşüren diller" arasında sayılabilir. Bağlamın anlaşılabildiği durumlarda özne zamirinin veya nesne zamirinin tekrarlanmasına gerek duyulmaz».

8. Texte original en turc de la citation traduite: «Adıllar Fransızca öğretimininde önemli bir yere sahiptir. Özne durumundaki adılların asla silinemeyeceği ögrenenlere öğretmenler tarafından anlatılmalıdır».

9. Texte original en turc de la citation traduite: «Türkçe’nin sözdiziminde eksiltili anlatımlar oldukça önemli bir yer tutar. Özellikle gizli özneli tümceler, başka birçok dilde bulunmayan, Türkçe’ye özgü anlatım özellikleridir. [...]. Fransızca bu türden kullanımlara izin vermez. Kurallı bir tümcede özne zorunludur ve tümcenin başında yer alır».

10. «bize attıkları oklar»: telle est traduite l'expression vers le turc, par Semih Atayman 2006, dans Sefiller, Istanbul: éditions Bordo-Siyah (ISBN 975-8688-51-0/975-8688-52-9).

11. Texte original en turc de la citation traduite: «Deyimler, atasözleri ve ikilemeler Türkçenin söz varlığı içerisinde önemli bir yere sahiptir. Örneklerden de anlaşılacağı üzere Türk dilinin tarihsel süreci boyunca; çalışma konusu söz öbekleri Türkçe söz varlığının içinde hep var olmuş; Türk dilini güçlendirmiş, akıcı, kıvrak ve derinlikli bir anlatımın yapı taşları olmuştur».

12. SenemoĞLu, Osman (24 juin 2013): La conquête de la langue ou identité et traduction: l'exemple turc. Fabula: la recherche en littérature. Consulté le $1^{\text {er }}$ février 2019, <http://www.fabula.org/ colloques/document1982.php>.

13. Texte original en en turc de la citation traduite: «Türkçe'de dolambaçlı birleşik cümleler, devrik cümlelerin kullanımı çok azdır. Soyut kavramlar, somut sözcüklerle anlatılır».

14. Texte original de la citation traduite: «La traduzione, ed e principio ormai ovvio in traduttologia, non avviene tra sistemi, bensi tra testi».

15. * Ünlü bir Fransız hakimi. Paris Yüksek Mahkemesi'nin ilk Başkanı (L'explication en turc du personnage Lamoignon)

16. ${ }^{* *}$ Fransız bahçe ve park desinatörü (1613-1700) (L'explication en turc du personnage Lenôtre)

\section{RÉFÉRENCES}

Akyalçın, Necmi et Gürcü, Hacer (2017): Orhan Pamuk ve Fakir Bayburt'un iki romanında deyim, atasözü ve ikilemeler bakımından söz varlığı [Existence du trésor langagier sur le plan des anaphores, proverbes et locutions dans les romans d'Orhan Pamuk et Fakir Bayburt]. Journal of Awareness. 2(1):55-68.

Alan, Sebahat (2005): Fransızca öğrenen Türklerin yaptıkları biçimbilimsel ve sözdizimsel yanlışların çözümlenmesi [Analyse des erreurs morphologiques et syntaxiques des Turcs apprenant le français]. Thèse de doctorat, non publiée. Ankara: Ankara Universitési Sosyal Bilimler Enstitüsü.

Andújar Moreno, Gemma (2013): Texte et paratexte dans la traduction assermentée: une approche contrastive français-espagnol. Meta. 58(1):66-86.

Ballard, Michel (2006): A propos des procédés de traduction. In: Christine Raguet, dir. Traduire ou «Vouloir garder un peu de la poussière d'or». Palimpsestes. Hors-série (2006):113-130.

Berman, Antoine et Berner, Christian (1999): Le penchant à traduire. La vraie traduction. In: Friedrich Schleiermacher. Des différentes méthodes du traduire: et autre texte. (Traduit de l'allemand par Antoine Berman et Christian Berner) Paris: Seuil, 11-26. 
Blanchet, Philippe (1995): La pragmatique d'Austin à Goffman. Paris: Bertrand-Lacoste.

BORDET, Geneviève (2014): Quand traduire, c'est décider: ce que l'interprétation des régularités statistiques d'un corpus peut apporter à la traduction spécialisée. ASp. 66:103-119.

Chesterman, Andrew (1997): Memes of Translation: The spread of ideas in translation theory. Amsterdam/Philadelphie: John Benjamins.

Chuquet, Hélène et Paillard, Michel (1987): Approches linguistiques des problèmes de traduction anglais-français. Paris: Ophrys.

Clas, André (2003): Compte rendu de Memes of Translation par Andrew Chesterman [2000, Amsterdam/Philadelphie: John Benjamins]. Meta. 48(4):607-611.

Conblin, Francis (1995): Les formes de reprise dans le discours: Anaphores et chaînes de référence. Rennes: Presses universitaires de Rennes.

Delisle, Jean (1980): L'analyse du discours comme méthode de traduction: initiation à la traduction française de textes pragmatiques en anglais: théorie et pratique. Ottawa: Presses universitaires de l'Université Ottawa.

Delisle, Jean (2003): La traduction raisonnée. Ottawa: Presses universitaires de l'Université Ottawa.

DelisLe, Jean (2005): L'enseignement pratique de la traduction. Ottawa: Presses universitaires de l'Université Ottawa.

Eco, Umberto (2003): Dire quasi la stessa cosa. Esperienze di traduzione [Dire presque la même chose. Expériences de traduction]. Milan: RCS Libri S.p.A.

Guidère, Mathieu (2008): Introduction à la traductologie. Penser la traduction, hier, aujourd'hui, demain. Bruxelles: De Boeck Supérieur.

Guidère, Mathieu (2010): Introduction à la théorie analytique de la traduction et de l'interprétation. Babel. 56(4):299-312.

Hiernard, Jean-Marc (2003): Les règles d'or de la traduction. Paris: Ellipses Edition.

KLEIBER, Georges (1986): Déictique, embrayeurs, «token-reflexives», symboles indexicaux, etc. comment les définir? L'information grammaticale. 30:3-22.

Kleiber, Georges (1989): Référence, texte et embrayeurs. Semen: Revue sémiolinguistique des textes et discours. 4: paragraphes 22-23. Consulté le 3 mars 2019, <https://journals.openedition.org/semen/6813>.

KLeIber, Georges (1994). Anaphores et pronoms. Louvain-la-Neuve: Duculot.

KLEIBER, Georges (1990): Marqueurs référentiels et processus interprétatifs: pour une approche plus sémantique. Cahiers de linguistique française. 11:241-258.

Konmuş, Sevtap (2007): Türkçe'de Franz Kafka ve Dönüşüm. Çeviribilimsel Karşılaştırmalı bir inceleme [Franz Kafka et la Métamorphose en turc. Une analyse comparative traductologique]. Mémoire de maîtrise, non publié. Ankara: Ankara Üniversitesi Sosyal Bilimler Enstitüsü.

KoRKuT, Ece (2008): La pragmatique et l'implicite. Synergies Turquie. 1:153-159.

KösE, Bayram (2011): Fransızcadan Türkçeye çeviride kendi zamiri [Le pronom «kendi» dans la traduction français-turc]. In: I. Uluslararası Çeviribilim ve Terimbilim kurultayı, Bildiri kitabı [Actes du premier congrès international sur la traductologie et la terminologie]. (I. Uluslararası Çeviribilim ve Terimbilim Kurultayı [Premier congrès international sur la traductologie et la terminologie], Kırıkkale, 20-21 octobre 2011). Kırıkkale: Université Kirikkale, 283-293.

KRINGS, Hans-Peter (1986): Was in den Köpfen von Übersetzern vorgeht. Eine empirische Untersuchung zur Struktur des Übersetzungsprozesses an fortgeschrittenen Französischlernen [Ce qui se passe dans l'esprit des traducteurs. Une étude empirique sur la structure du processus de traduction dans l'apprentissage du français avancé]. Tubingue: Narr.

KüNZLI, Alexandre (2003): Quelques stratégies et principes en traduction technique françaisallemand et français-suédois. Thèse de doctorat, non publiée. Stockholm: Akademitryck.

Ladmiral, Jean-René (1979): Traduire: Théorèmes pour la traduction. Paris: Payot.

LAdMIRAL, Jean-René (1979/1994): Traduire: Théorèmes pour la traduction. $2^{\mathrm{e}}$ éd. Paris: Gallimard. 
Larose, Robert (1989): Théories contemporaines de la traduction. Québec: Presses de l'Université du Québec.

Lederer, Marianne (1994): La traduction aujourd'hui. Paris: Hachette.

Özçelik, Nurten (2008): Causes des difficultés de l'écrit en français chez les étudiants turcs. Gazi Ĕ̆itim Fakültesi Dergisi. 28(3):99-123.

Perdicoyanni-Paléologou, Hélène (2001): Le concept d'anaphore, de cataphore et de déixis en linguistique française. Revue québécoise de linguistique. 29(2):55-77.

Poncharal, Bruno (2010): La traduction de l'anaphore dans la prose de pensée. Palimpsestes. 23:41-62.

Riceur, Paul (1965): De l'interprétation. Paris: Seuil.

Riceur, Paul (2004): Sur la traduction. Paris: Bayard.

Schleiermacher, Antoine (1813/1999): Des différentes méthodes du traduire: et autre texte. (Traduit de l'allemand par Antoine Berman et Christian Berner). Paris: Seuil.

Seleskovitch, Danica et Lederer, Marianne (2001): Interpréter pour traduire. Paris: Didier Érudition (Klincksieck).

ŞıмşEK, Rasim (1987) : Örneklerle Türkçe Sözdizimi. Tümceler-Belirtme Öbekleri- Çözümleme [Exemples de la syntaxe du turc et phrases, groupes de description et analyse] Trabzon: Kuzey Matbaacilık.

Szlamowicz, Jean (2011) : L'écart et l'entre-deux: traduire la culture. Sillages critiques. 12:21 p. Consulté le 23 novembre 2018, <https://journals.openedition.org/sillagescritiques/2314>.

VAn Hoof, Henri (1989): Traduire l'anglais. Théorie et pratique. Louvain-la-Neuve: Duculot.

Vinay, Jean-Paul et Darbelnet, Jean (1958/1966): Stylistique comparée du français et de l'anglais. Paris: Didier.

VReCK, Françoise (1992): ABC de la version anglaise. Paris: Longman France.

Widlund-Fantini, Anne-Marie (2007): Danica Seleskovitch interprète et témoin du XXé siècle. Paris: Broché

Wolfram, Walt (2006): Variation in language: overview. In: Keith Brown, dir. Encyclopedia of languages and linguistics. $2^{\mathrm{e}}$ éd. Oxford: Elsevier, 333-340.

YILANCIOĞLU, Seza (2005): Türk romanının oluşumunda çevirinin ekinsel etkileri [Les effets culturels de la traduction dans la formation du roman turc]. In: Ali Osman ÖzTürK, Nevide Akpinar-Dellal et Umut Balci, dir. IV. Uluslararası Dil, Yazın ve Deyişbilim Sempozyumu Bildirileri [Actes du $4^{e}$ symposium international sur la langue, la littérature et les dictons]. (Uluslararası IV. Dil, Yazın ve Deyişbilim Sempozyumu [4 ${ }^{\mathrm{e}}$ symposium international sur la langue, la littérature et les dictons], Çanakkale, 17-19 juin 2005). Çanakkale: Ç.O.M.Ü. Yayınları, 287-296. 